\title{
The Essential Spectrum of the Laplacian on Manifolds with Ends
}

\author{
By
}

Toshiaki HATTORI*

\begin{abstract}
Let $V$ be a noncompact complete Riemannian manifold. We find a geometric condition which assures that the essential spectrum of the Laplacian on $V$ contains a half-line, by means of fiber bundle structures and the asymptotic behavior of mean curvatures on the ends of $V$, and give lower bounds of the essential spectrum. Our criteria can be applied to locally symmetric spaces of finite volume and manifolds of infinite volume canonically obtained from manifolds with corners.
\end{abstract}

\section{Introduction}

Let $V$ be an $n$-dimensional complete Riemannian manifold. When $V$ is noncompact, it is possible that the essential spectrum of the Laplacian on $V$ is nonempty. For example, it is well-known that in the case of $n$-dimensional complete hyperbolic manifolds of finite volume, the essential spectrum is the half-line $\left[(n-1)^{2} / 4, \infty\right)$. In the case of locally symmetric spaces of finite volume, the essential spectrum is known to be a half-line. In this paper we find a geometric condition which assures that the essential spectrum contains a halfline, by means of fiber bundle structures and the asymptotic behavior of mean curvatures on the ends of $V$, and give lower bounds of the essential spectrum under some additional condition. It is also our hope to understand the case of locally symmetric spaces of finite volume from a different point of view from Langlands' theory of Eisenstein series ([20], [25]), and investigate manifolds of infinite volume canonically obtained from manifolds with corners.

\footnotetext{
Communicated by S. Mukai. Received July 3, 2006. Revised July 9, 2007.

2000 Mathematics Subject Classification(s): Primary 58J50; Secondary 35P15.

* Department of Mathematics, Tokyo Institute of Technology, Oh-okayama, Meguro, Tokyo 152-8551, Japan.

e-mail: hattori@math.titech.ac.jp
}

(c) 2009 Research Institute for Mathematical Sciences, Kyoto University. All rights reserved. 
For studying the essential spectrum of $V$, there is a known method, referred to as the decomposition principle $([10],[15])$ : The essential spectrum is stable under compact perturbations. Thus this spectrum does not change when one modifies the manifold in a compact region. Hence, we are motivated by the following question: What kind of geometric or metric structure of the ends produces a half-line in the essential spectrum ?

First we consider a simple example. Let $M$ be a compact manifold with a connected boundary $\partial M$. Then we can attach a half-cylinder $\partial M \times[0, \infty)$ to the boundary to produce a complete Riemannian manifold $V$. If the halfcylinder is equipped with the product metric $g+d t^{2}$, where $g$ is a metric on $\partial M$ and $d t^{2}$ is the standard metric on $[0, \infty)$, the essential spectrum equals $[0, \infty)$. When $\partial M$ is the flat torus and the half-cylinder is equipped with a warped product metric $e^{-2 t} g+d t^{2}$, the essential spectrum equals $\left[(n-1)^{2} / 4, \infty\right)$. This corresponds to the case of $n$-dimensional hyperbolic manifolds of finite volume. Therefore we next consider metrics which are not necessarily warped products.

Let $Y_{1}, \ldots, Y_{s}$ be closed manifolds. Let $\phi_{j}: Y_{j} \times[0, \infty) \longrightarrow V$ be an embedding and $\mathcal{E}_{j}=\phi_{j}\left(Y_{j} \times(0, \infty)\right)$ for each $j=1, \ldots, s$. Suppose that $V-\bigcup_{j=1}^{s} \mathcal{E}_{j}$ is a compact submanifold with boundary. In particular, $V$ is a manifold with $s$ ends. We suppose that the induced metric on $Y_{j} \times[0, \infty)$ through $\phi_{j}$ is of the form $g_{j, t}+d t^{2}$, where $g_{j, t}$ is a metric on $Y_{j}$ depending on $t \geq 0$. Let $\sqrt{g_{j, t}}(y)$ be the square root of the determinant of the metric tensor of $g_{j, t}$ at $y \in Y_{j}$. We also suppose that

$$
\text { the ratio } \alpha_{j}(t):=\sqrt{g_{j, t}}(y) / \sqrt{g_{j, 0}}(y) \text { does not depend on } y
$$

on each $Y_{j} \times[0, \infty)$. In this paper, we call such a manifold $V$ a Riemannian manifold with boundaries $Y_{j}$ at infinity. This class of manifolds contains $\mathbb{R}$ rank 1 or $\mathbb{Q}$-rank 1 locally symmetric spaces of finite volume. However, we do not necessarily assume that $V$ is nonpositively curved or of finite volume. If $Y_{j}$ has a structure of some special fiber bundle, it might give rise to some additional structure on the essential spectrum. Because of this and the fact that the end of any higher $\mathbb{Q}$-rank locally symmetric space of finite volume is more complicated, we also consider the following situation.

Let $B$ be a manifold (without boundary) and $g_{B}$ a complete Riemannian metric on $B$. Let $\pi: Y \longrightarrow B$ be a fiber bundle with compact fibers. We suppose that the dimension of $Y$ is $n-d$ with $d \geq 1$. Let $\mathcal{C} \subset \mathbb{R}^{d}$ be an open (infinite) cone, that is, a region enclosed by $d$ hyperplanes, and let $d \mathbf{t}^{2}$ be the standard metric of $\mathbb{R}^{d}$. We suppose that there exists an open embedding $\phi: Y \times \mathcal{C} \longrightarrow V$ and that the induced metric on $Y \times \mathcal{C}$ is of the form $g_{\mathbf{t}}+d \mathbf{t}^{2}$. 
Here $\left\{g_{\mathbf{t}}\right\}_{\mathbf{t} \in \mathcal{C}}$ is a family of complete Riemannian metrics on $Y$ of the form

$$
g_{\mathbf{t}}(y)=g_{\mathbf{t}}^{\perp}(y)+\left(\pi^{*} g_{B}\right)(y),
$$

where $g_{\mathbf{t}}^{\perp}(y)$ is a metric on the tangent space $E_{y}$ of the fiber $\pi^{-1}(\pi(y))$ at $y$ depending on $\mathbf{t}$, and $\left(\pi^{*} g_{B}\right)(y)$ is the metric on the orthogonal complement of $E_{y}$ in the tangent space $T_{y}(Y)$ of $Y$ at $y$. In particular, this means that $\pi:\left(Y, g_{\mathbf{t}}\right) \longrightarrow\left(B, g_{B}\right)$ is a Riemannian submersion. Let $l:[0, \infty) \longrightarrow \mathbb{R}^{d}$ be a ray contained in the interior of $\mathcal{C}$. In the case $d \geq 2$ we suppose that $l$ is not parallel to any of the boundary hyperplanes of $\mathcal{C}$. We denote by $\mathcal{C}_{t}$ the hyperplane in $\mathcal{C}$ through $l(t)$ orthogonal to $l$. Let $\left(t, t_{2}, \ldots, t_{d}\right)$ be a Cartesian coordinate system of $\mathbb{R}^{d}$ such that the origin $\mathbf{0}$ is $l(0)$ and that the positive part of the $t$-axis corresponds to the ray $l$.

Let $\sqrt{g_{\mathbf{t}}}(y)$ be the square root of the determinant of the metric tensor of $g_{\mathbf{t}}$ at $y \in Y$. We consider the following two conditions.

The ratio $\sqrt{g_{\mathbf{t}}}(y) / \sqrt{g_{\mathbf{0}}}(y)$ depends only on $t$.

The projection $\pi$ is harmonic with respect to the metric $g_{\mathbf{t}}$.

These two conditions $(*),(* *)$ are satisfied in the case of locally symmetric spaces of finite volume. We explain the higher $\mathbb{Q}$-rank case in later sections. In this paper we suppose that the condition $(*)$ is always satisfied unless otherwise mentioned, and denote the ratio by $\alpha(t)$ :

$$
\alpha(t)=\sqrt{g_{\mathbf{t}}}(y) / \sqrt{g_{\mathbf{0}}}(y)
$$

We do not necessarily assume the condition $(* *)$.

Let

$$
\beta(t)=\frac{1}{2} \log \alpha(t)
$$

As we show in Section 1, the mean curvature of $Y \times \mathcal{C}_{t}$ in $Y \times \mathcal{C}$ depends not on $y \in Y$ but on $t$, and is equal to

$$
-\frac{1}{n-1} \frac{\alpha^{\prime}(t)}{\alpha(t)}=-\frac{2}{n-1} \beta^{\prime}(t)
$$

We denote this by $\mathcal{K}(t)$. Let $\bar{\Delta}$ be the unique self-adjoint extension ([8], [13]) of the Laplacian $\Delta$ on $V$ to the Hilbert space $L^{2}(V)$ of square integrable (complexvalued) functions on $V$, and $\bar{\Delta}_{B}$ the similar extension of the Laplacian $\Delta_{B}$ on $B$. 
Theorem 1. Suppose that

$$
\lim _{t \rightarrow \infty}\left\{\mathcal{K}(t)^{2}-\frac{2}{n-1} \mathcal{K}^{\prime}(t)\right\}=\kappa^{2}
$$

exists.

(1) If $B$ is compact, then for any $r \geq 0$, there exists a family $\left\{u_{r, \varepsilon}\right\}_{\varepsilon>0}$ of compactly supported smooth functions on $\mathcal{E}:=\phi(Y \times \mathcal{C})$ satisfying the following two conditions.

(0.1) For any compact subset of $\mathcal{E}$, if we take $\varepsilon$ sufficiently small, then the support of $u_{r, \varepsilon}$ lies outside this compact set.

(0.2) For some positive constant $C_{1}$ independent of $\varepsilon$, we have

$$
\left\|\left(\Delta-\left(\frac{(n-1)^{2} \kappa^{2}}{4}+r^{2}\right)\right) u_{r, \varepsilon}\right\| \leq C_{1} \varepsilon\left\|u_{r, \varepsilon}\right\|,
$$

where \|\| is the $L^{2}$-norm on $L^{2}(V)$. $\bar{\Delta}$.

In particular, every point of $\left[(n-1)^{2} \kappa^{2} / 4, \infty\right)$ belongs to the spectrum of

(2) Suppose that the condition $(* *)$ is satisfied. If $\bar{\Delta}_{B}$ has a sequence

$$
c_{0}<c_{1}<\cdots<c_{m}<\cdots
$$

of eigenvalues, then the following holds.

For each $m$ and any $r \geq 0$, there exists a family $\left\{u_{m, r, \varepsilon}\right\}_{\varepsilon>0}$ of compactly supported smooth functions on $\mathcal{E}=\phi(Y \times \mathcal{C})$ satisfying the following three conditions.

(0.3) For any compact subset of $\mathcal{E}$, if we take $\varepsilon$ sufficiently small, then the support of $u_{m, r, \varepsilon}$ lies outside this compact set.

(0.4) For some positive constant $C_{1}$ independent of $\varepsilon$, we have

$$
\left\|\left(\Delta-\left(\frac{(n-1)^{2} \kappa^{2}}{4}+c_{m}+r^{2}\right)\right) u_{m, r, \varepsilon}\right\| \leq C_{1} \varepsilon\left\|u_{m, r, \varepsilon}\right\| .
$$

(0.5) If $m \neq m^{\prime}$, then

$$
\lim _{\substack{\varepsilon, 0 \\ \varepsilon^{\prime} \longrightarrow 0}}\left(u_{m, r, \varepsilon}, u_{m^{\prime}, r^{\prime}, \varepsilon^{\prime}}\right)=0
$$

where $\left(\right.$, ) is the $L^{2}$-inner product on $L^{2}(V)$. of $\bar{\Delta}$.

In particular, every point of $\left[(n-1)^{2} \kappa^{2} / 4+c_{0}, \infty\right)$ belongs to the spectrum 
Remark. In the case (2), if a non-negative number $c$ belongs to the essential spectrum of $\bar{\Delta}_{B}$, then we can construct for any $r \geq 0$ a family $\left\{u_{r, \varepsilon}\right\}_{\varepsilon>0}$ of compactly supported smooth functions on $\mathcal{E}$ such that the same condition as (0.3) and the inequality

$$
\left\|\left(\Delta-\left(\frac{(n-1)^{2} \kappa^{2}}{4}+c+r^{2}\right)\right) u_{r, \varepsilon}\right\| \leq C_{1} \varepsilon\left\|u_{r, \varepsilon}\right\|
$$

are satisfied (cf. Proposition 1.2).

Remark. Although we followed the decomposition principle to obtain the statement of Theorem 1, we do not need to use it directly in the proof.

In order to give lower bounds of the essential spectrum, we can use the following known result on Rayleigh quotients. Since there seem to be no suitable references, we also include its proof in Section 2 for convenience.

Lemma 1. Let $N$ be an open subset of a complete Riemannian manifold. Let $Z$ be a $C^{1}$-vector field on $N$ such that

(0.6) its norm $|Z|$ is bounded from above by some positive constant $C:|Z| \leq C$, and that

(0.7) the divergence of $Z$ is bounded away from zero: that is, $\operatorname{div} Z \geq \varepsilon>0$ for some $\varepsilon$.

Then we have

$$
\inf \frac{\int_{N}|\operatorname{grad} u|^{2} d \mu_{N}}{\int_{N}|u|^{2} d \mu_{N}} \geq\left(\frac{\varepsilon}{2 C}\right)^{2} \quad \text { on } N,
$$

where $\mu_{N}$ is the canonical measure on $N$ induced from the Riemannian metric and $u$ runs through all the compactly supported smooth functions on $N$.

We first restrict ourselves to the case of Riemannian manifolds with boundaries $Y_{j}$ at infinity. We denote by $\mathcal{K}_{j}(t)$ the mean curvature of $Y_{j} \times\{t\}$ in $Y_{j} \times(0, \infty)$. Applying Lemma 1 to the vector field $\frac{\partial}{\partial t}$ or $-\frac{\partial}{\partial t}$ on each end, we obtain the following immediately from the decomposition principle.

Proposition 1. Let $V$ be a Riemannian manifold with boundaries $Y_{j}$ at infinity. Suppose that $\lim _{t \rightarrow \infty} \mathcal{K}_{j}(t)=\kappa_{j}$ exists for each $j$. Let $\kappa^{2}=\min _{j}\left(\kappa_{j}\right)^{2}$. Then the essential spectrum of $\bar{\Delta}$ is contained in the interval $\left[(n-1)^{2} \kappa^{2} / 4, \infty\right)$.

We show the following theorem of different type.

Theorem 2. Let $V$ be a Riemannian manifold with boundaries $Y_{j}$ at infinity. Suppose that there exists a positive number a such that

$$
\mathcal{K}_{j}(t)^{2}-\frac{2}{n-1} \mathcal{K}_{j}^{\prime}(t) \geq a^{2} \quad \text { on }(0, \infty)
$$


for each $j$. We also suppose that for each $j$, there exist $C_{2}(j), C_{3}(j)$ such that

$$
\begin{gathered}
0<C_{2}(j)<C_{3}(j), \\
\mathcal{K}_{j}(t)>0 \text { on }\left[C_{2}(j), C_{3}(j)\right) .
\end{gathered}
$$

The numbers $C_{2}(j), C_{3}(j)$ may depend on $j$. Then, on the interval $[0,(n-$ $\left.1)^{2} a^{2} / 4\right)$ the spectrum of $\bar{\Delta}$ consists of at most a finite number of eigenvalues of finite multiplicity. If $(n-1)^{2} a^{2} / 4$ is an eigenvalue, then its multiplicity is finite.

These results give alternative proofs of some of the known results on rank 1 locally symmetric spaces. Let $G$ be a connected semisimple Lie group having finite center and no compact factors. Let $K$ be a maximal compact subgroup of $G$ and $X=G / K$ the associated symmetric space of noncompact type with the canonical left invariant metric. Let $\Gamma$ be a torsion-free irreducible non-uniform lattice of $G$ and $V=\Gamma \backslash X$. Let us call $V$ an $\mathbb{R}$-rank $k$ locally symmetric space of finite volume if $X$ is a rank $k$ symmetric space. We call $V$ a $\mathbb{Q}$-rank $k$ locally symmetric space of finite volume if $G$ has trivial center and is the identity component of the group of real points of some connected semisimple linear algebraic group $\mathbf{G}$ defined over $\mathbb{Q}$ of $\mathbb{Q}$-rank $k$ and if $\Gamma$ is an arithmetic subgroup of G. Let $\mathbb{R} \rho$ (resp. $\rho$ ) be the half sum of the positive roots (resp. $\mathbb{Q}$-roots). Then any $\mathbb{R}$-rank 1 (resp. $\mathbb{Q}$-rank 1 ) locally symmetric space of finite volume is a Riemannian manifold with boundaries $Y_{j}$ at infinity with $\mathcal{K}_{j}(t)=\left.2\right|_{\mathbb{R}} \rho \mid /(n-1)($ resp. $2|\rho| /(n-1))([14],[12],[4],[26]$, [5], see also [9], [23]). Hence we have

Theorem 3 (cf. [9], [23], [6]). Let $V$ be an $\mathbb{R}$-rank 1 (resp. a $\mathbb{Q}$-rank 1 ) locally symmetric space of finite volume. Then the essential spectrum of $\bar{\Delta}$ is the half-line $\left[\left.\left.\right|_{\mathbb{R}} \rho\right|^{2}, \infty\right)\left(\right.$ resp. $\left.\left[|\rho|^{2}, \infty\right)\right)$. If $|\mathbb{R} \rho|^{2}\left(\right.$ resp. $\left.|\rho|^{2}\right)$ is an eigenvalue, then its multiplicity is finite.

Remark. Let $V$ be a $\mathbb{Q}$-rank $1, \mathbb{R}$-rank $\geq 2$ locally symmetric space of finite volume. Then each boundary $Y_{j}$ at infinity admits a fiber bundle structure $\pi_{j}: Y_{j} \longrightarrow B_{j}$ satisfying the conditions $(\star \star),(* *)$ (see Remark before Theorem 4 in Section 3). Let $\bar{\Delta}_{B_{j}}$ be the unique self-adjoint extension of the Laplacian on the base space $B_{j}$ of $Y_{j}$. Then it follows from Theorem 1 that for each eigenvalue $c$ of $\bar{\Delta}_{B_{j}}$, there exists a family of compactly supported smooth functions on $V$ which assures that the half-line $\left[|\rho|^{2}+c, \infty\right)$ is contained in the essential spectrum of $\bar{\Delta}$. 
In the case where $V$ is a higher $\mathbb{Q}$-rank locally symmetric spaces of finite volume, there is a compactification $\bar{V}$ of $V([7])$ such that $\bar{V}$ is a manifold with corners and its boundary $\partial \bar{V}$ is connected. Each stratum $Y_{j}$ of $\partial \bar{V}$ admits a fiber bundle structure $\pi_{j}: V_{j} \longrightarrow B_{j}$ satisfying the conditions $(\star \star),(* *)$. The continuous spectrum of $V$ is controlled by the eigenvalues of the base spaces $B_{j}$ : For each eigenspace of $B_{j}$ with eigenvalue $c$, there exists a certain subspace of $L^{2}(V)$ corresponding to the continuous spectrum. The space $L^{2}(V)$ is the closure of the union of such subspaces and the eigenspaces corresponding to the point spectrum of $V$. This follows from Langlands' spectral resolution of the regular representation of $G$ on $L^{2}(\Gamma \backslash G)([20],[25])$. Although the situation is slightly different from the one in Theorem 1, the argument in the proof of Theorem 1 can be also applied to this case and we obtain a similar result (Theorem 4) to Theorem 3 by constructing a vector field as in Lemma 1 . Thus we can give alternative proofs of some of the above facts without using Langlands' theory of Eisenstein series. We postpone describing Theorem 4 until Section 3, since we need more notations.

Theorem 1 can be applied to complete manifolds canonically obtained from manifolds with corners as in Figure 7 (see Section 5 for the precise definition).

Corollary 1. Let $W$ be a manifold with compact corners and $V$ the complete manifold obtained from $W$ by gluing cylinders successively to boundary components. Then the essential spectrum of $V$ is the half-line $[0, \infty)$.

Our calculations in the proof of Theorems 1 and 2 are based on higher dimensional generalizations of Lax-Phillips' ones $([21, \S 4])$. In order to construct the sequences $\left\{u_{m, r, \varepsilon}\right\}_{\varepsilon>0}$ in Theorem 1, under the identification of $\mathcal{E}$ with $Y \times \mathcal{C}$, we first consider the function $f_{0}(y, \mathbf{t})=\alpha(t)$ on $\mathcal{E}$ and multiply $f_{0}$ by an oscillation to obtain $f_{r}$. We take a product of $f_{r}$ with an eigenfunction $\varphi$ of $B$ with eigenvalue $c_{m}$ and control the support of this function by using a suitable cut-off function $h$ (see Figure 1). In particular, in the case of locally symmetric spaces of finite volume the function $f_{0}$ is induced from the exponential of a constant multiple of the Busemann function on $X$ with respect to some geodesic $\gamma:[0, \infty) \longrightarrow X$ which is projected on $l$.

This paper is organized as follows. In Section 1 we first show the explicit relation between the functions $\alpha(t), \beta(t)$, and the mean curvature $\mathcal{K}(t)$. Then we prove Theorem 1. In Section 2 we prove Theorems 2. In Sections 3 and 4 we explain the case of higher $\mathbb{Q}$-rank locally symmetric spaces. In the last section we discuss some consequences of our theorems including Corollary 1.

As usual we denote by $\mathbb{C}, \mathbb{R}, \mathbb{Q}, \mathbb{N}$ the set of the complex numbers, the real numbers, the rational numbers, the natural numbers, respectively. 


\section{$\S 1$. The Existence of a Half-Line in the Essential Spectrum}

In this section we prove Theorem 1 . We identify $\mathcal{E}$ with $Y \times \mathcal{C}$ by the diffeomorphism $\phi$. First we show that the mean curvature of $Y \times \mathcal{C}_{t}$ in $Y \times \mathcal{C}$ depends only on $t$.

Let $\mathbf{t}=\left(t, t_{2}, \ldots, t_{d}\right)$ be a Cartesian coordinate system of $\mathbb{R}^{d}$ such that the positive part of the $t$-axis corresponds to the ray $l$. We define a unit vector field $\xi$ on $Y \times \mathcal{C}$ by $\xi=\frac{\partial}{\partial t}$, which is orthogonal to $Y \times \mathcal{C}_{t}$ for each $t \geq 0$. We denote by $T_{P}\left(Y \times \mathcal{C}_{t}\right)$ the tangent space of $Y \times \mathcal{C}_{t}$ at $P \in Y \times \mathcal{C}_{t}$.

Definition 1.1. For each point $P$ of $Y \times \mathcal{C}_{t}$, let $A_{\xi}: T_{P}\left(Y \times \mathcal{C}_{t}\right) \longrightarrow$ $T_{P}\left(Y \times \mathcal{C}_{t}\right)$ be the shape operator with respect to $\xi$ defined by

$$
A_{\xi}(X)=-\nabla_{X} \xi \quad \text { for each } X \in T_{P}\left(Y \times \mathcal{C}_{t}\right),
$$

where $\nabla$ is the covariant derivative on $V$. We define the mean curvature $\mathcal{K}(P)$ of $Y \times \mathcal{C}_{t}$ at $P$ (with respect to $\xi$ ) by

$$
\mathcal{K}(P)=\frac{1}{n-1} \operatorname{trace}\left(A_{\xi}\right) .
$$

Proposition 1.1. We have

$$
\mathcal{K}(P)=-\frac{1}{n-1} \frac{\alpha^{\prime}(t)}{\alpha(t)}=-\frac{2}{n-1} \beta^{\prime}(t)
$$

for all $P \in Y \times \mathcal{C}_{t}$.

Proof. Let $P=\left(y^{*},\left(t^{*}, t_{2}{ }^{*}, \ldots, t_{d}{ }^{*}\right)\right) \in Y \times \mathcal{C}_{t^{*}}$. Take an arbitrary coordinate neighborhood $\left(U,\left(y_{1}, \ldots, y_{n-d}\right)\right)$ of $y^{*}$ in $Y$. We put $y_{n-d+j}=t_{j+1}$ for $j=1, \ldots, d-1$, and $y_{n}=t$ to obtain a local coordinate system $\left(y_{1}, \ldots, y_{n}\right)$ around $P$ in $Y \times \mathcal{C}_{t}$. Let $g_{j k}$ be the components of the metric tensor with respect to this local coordinate system, $\left(g^{j k}\right)=\left(g_{j k}\right)^{-1}$, and $\Gamma_{j k}^{m}$ the Christoffel symbols. We have

$$
\begin{aligned}
(n-1) \mathcal{K}(P) & =-\sum_{j=1}^{n-1} \Gamma_{j n}^{j}=-\frac{1}{2} \sum_{j=1}^{n-1} \sum_{m=1}^{n} g^{j m}\left(\frac{\partial g_{n m}}{\partial y_{j}}+\frac{\partial g_{j m}}{\partial y_{n}}-\frac{\partial g_{j n}}{\partial y_{m}}\right) \\
& =-\frac{1}{2} \sum_{j=1}^{n-1} \sum_{m=1}^{n-1} g^{j m}\left(\frac{\partial g_{j m}}{\partial t}\right) .
\end{aligned}
$$

Let $A=\left(g_{j k}\right)_{1 \leq j, k \leq n-1}$. Then we have

$$
(n-1) \mathcal{K}(P)=-\frac{1}{2} \operatorname{trace}\left(A^{-1} \frac{\partial A}{\partial t}\right)=-\frac{1}{2} \frac{\frac{\partial}{\partial t}(\operatorname{det} A)}{\operatorname{det} A}=-\frac{1}{2} \frac{\partial}{\partial t}(\log (\operatorname{det} A)) .
$$




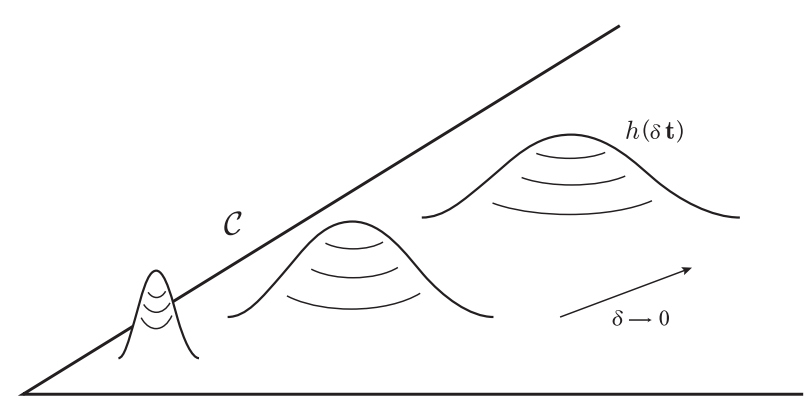

Figure 1.

Since the determinant of $A$ depends only on $t$ and is equal to $\left(\sqrt{g_{0}}\right)^{2} \alpha(t)^{2}$, we obtain

$$
(n-1) \mathcal{K}(P)=-(\log \alpha)^{\prime}\left(t^{*}\right)=-2 \beta^{\prime}\left(t^{*}\right) .
$$

The mean curvature $\mathcal{K}(P)$ is constant on each $Y \times \mathcal{C}_{t}$, which we denote by $\mathcal{K}(t)$.

Let $C_{0}^{\infty}(Y \times \mathcal{C})$ be the set of compactly supported smooth functions on $Y \times \mathcal{C}$. We define a map $T: C_{0}^{\infty}(Y \times \mathcal{C}) \longrightarrow C_{0}^{\infty}(Y \times \mathcal{C})$ by

$$
(T(f))(y, \mathbf{t})=f(y, \mathbf{t}) / \sqrt{\alpha(t)}
$$

for $f \in C_{0}^{\infty}(Y \times \mathcal{C})$. Then we have

$$
T^{-1} \circ \Delta \circ T=-\frac{\partial^{2}}{\partial t^{2}}+\frac{(n-1)^{2}}{4}\left\{\mathcal{K}(t)^{2}-\frac{2}{n-1} \mathcal{K}^{\prime}(t)\right\}-\sum_{j=2}^{d} \frac{\partial^{2}}{\partial t_{j}^{2}}+\Delta_{\left(Y, g_{\mathbf{t}}\right)}
$$

on $C_{0}^{\infty}(Y \times \mathcal{C})$, where $\Delta_{\left(Y, g_{\mathbf{t}}\right)}$ is the Laplacian on $\left(Y, g_{\mathbf{t}}\right)$. Let $\left(\tau_{\mathbf{t}}\right)_{y}$ be the trace of the second fundamental form of the submanifold $\pi^{-1}(\pi(y))$ of $\left(Y, g_{\mathbf{t}}\right)$ at $y \in Y$, and $C_{0}^{\infty}(B)$ the set of compactly supported smooth functions on $B$. From the formula on Riemannian submersion in Theorem 4.4 of [19, XIV, §4], we have

$$
\Delta_{\left(Y, g_{\mathbf{t}}\right)}(\varphi \circ \pi)(y)=\left(\Delta_{B} \varphi\right)(\pi(y))+\left(\tau_{\mathbf{t}}\right)_{y} \cdot(\varphi \circ \pi) \quad \text { for all } \varphi \in C_{0}^{\infty}(B)
$$

Let $f_{0}$ be the function on $Y \times \mathcal{C}$ defined by

$$
f_{0}(y, \mathbf{t})=e^{-\beta(t)}=\frac{1}{\sqrt{\alpha(t)}} .
$$




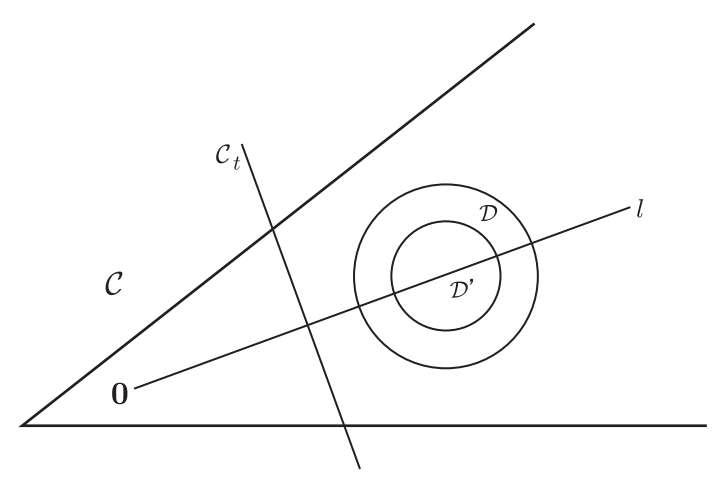

Figure 2.

For each $r \geq 0$, we define a function $f_{r}$ by

$$
f_{r}(y, \mathbf{t})=e^{-\beta(t)+\sqrt{-1} \cdot r t} .
$$

For $\varphi \in C_{0}^{\infty}(B)$, we put

$$
f_{\varphi, r}(y, \mathbf{t})=e^{-\beta(t)+\sqrt{-1} \cdot r t} \varphi(\pi(y)) .
$$

Let $\mathcal{D}$ and $\mathcal{D}^{\prime}$ be open balls in $\mathbb{R}^{d}$ with the same center on the ray $l$ such that $\mathcal{D}^{\prime} \subset \mathcal{D} \subset \mathcal{C}$ and that $\mathbf{0} \notin \mathcal{D}$, where $\mathbf{0}$ is the origin of $\mathbb{R}^{d}$. We choose $\mathcal{D}$ such that every ray emanating from the origin which is tangent to the sphere bounding $\mathcal{D}$ is entirely contained in $\mathcal{C}$. We also suppose that

$$
\left(\bigcup_{0 \leq t<C_{4}} \mathcal{C}_{t}\right) \cap \mathcal{D}=\phi
$$

for a positive number $C_{4}$ (see Figure 2). Let $h: \mathbb{R}^{d} \longrightarrow[0,1]$ be a smooth function such that $h>0$ on $\mathcal{D}, h \equiv 0$ outside $\mathcal{D}$, and $h \equiv 1$ on the closure of $\mathcal{D}^{\prime}$. by

For any positive number $\delta \leq 1$, we define a smooth function $f_{\varphi, r, \delta}$ on $Y \times \mathcal{C}$

$$
f_{\varphi, r, \delta}(y, \mathbf{t})=h(\delta \mathbf{t}) e^{-\beta(t)+\sqrt{-1} \cdot r t} \varphi(\pi(y)) .
$$

Let $L^{2}(B)$ be the Hilbert space of square integrable functions on $B$ with $L^{2}$ norm \|\|$_{B}$.

Proposition 1.2. Suppose that $\left(\tau_{\mathbf{t}}\right)_{y} \cdot(\varphi \circ \pi)=0$ for any $y \in Y, \mathbf{t}=$ $\left(t, t_{2}, \ldots, t_{d}\right) \in \mathcal{C}$ with $t \geq 0$, and that $\lim _{t \rightarrow \infty}\left\{\mathcal{K}(t)^{2}-2 \mathcal{K}^{\prime}(t) /(n-1)\right\}=\kappa^{2}$. 
Then for any $\varepsilon>0$, there exists a positive number $\delta(\varepsilon)$ such that the following holds.

Let $\delta$ be a positive number smaller than $\delta(\varepsilon), c \geq 0$, and $\varphi \in C_{0}^{\infty}(B)$ with

$$
\left\|\left(\Delta_{B}-c\right) \varphi\right\|_{B}<\varepsilon\|\varphi\|_{B} .
$$

Then we have

$$
\left\|\left(\Delta-\left(\frac{(n-1)^{2} \kappa^{2}}{4}+c+r^{2}\right)\right) f_{\varphi, r, \delta}\right\|<C_{5} \varepsilon\left\|f_{\varphi, r, \delta}\right\|,
$$

where $C_{5}$ is a constant depending only on $h, r$, and $\varphi$.

We denote by $\mu, \mu_{Y}, \mu_{B}, \mu_{\mathbf{t}}$ the Riemannian measure of $V,\left(Y, g_{\mathbf{0}}\right),\left(B, g_{B}\right)$, $\mathcal{C}$, respectively, and $\mu_{Y, \mathbf{t}}=e^{2 \beta(t)} \mu_{Y}$ the Riemannian measure of $\left(Y, g_{\mathbf{t}}\right)$.

Proof. Let

$$
\begin{aligned}
& A_{1}(y, \mathbf{t})=\left\{\left(\beta^{\prime}(t)^{2}+\beta^{\prime \prime}(t)\right)-\frac{(n-1)^{2} \kappa^{2}}{4}\right\} f_{\varphi, r, \delta}(y, \mathbf{t}) \\
& -\delta\left\{2 \sqrt{-1} r \frac{\partial h}{\partial t}(\delta \mathbf{t})+\delta\left(\frac{\partial^{2} h}{\partial t^{2}}+\sum_{j=2}^{d} \frac{\partial^{2} h}{\partial t_{j}^{2}}\right)(\delta \mathbf{t})\right\} \varphi(\pi(y)) e^{-\beta(t)+\sqrt{-1} \cdot r t}
\end{aligned}
$$

and

$$
A_{2}(y, \mathbf{t})=\left(\Delta_{B}-c\right) \varphi(\pi(y)) h(\delta \mathbf{t}) e^{-\beta(t)+\sqrt{-1} \cdot r t} .
$$

Then, it follows from (1.1), (1.2) that

$$
\left(\Delta-\left(\frac{(n-1)^{2} \kappa^{2}}{4}+c+r^{2}\right)\right) f_{\varphi, r, \delta}(y, \mathbf{t})=A_{1}(y, \mathbf{t})+A_{2}(y, \mathbf{t}) .
$$

We have

$$
\begin{aligned}
\left|A_{1}(y, \mathbf{t})\right| & \leq\left|\frac{(n-1)^{2} \kappa^{2}}{4}-\left\{\left(\beta^{\prime}\right)^{2}+\beta^{\prime \prime}\right\}\right|\left|f_{\varphi, r, \delta}\right| \\
& +\delta\left|2 \sqrt{-1} r \frac{\partial h}{\partial t}(\delta \mathbf{t})+\delta\left(\frac{\partial^{2} h}{\partial t^{2}}+\sum_{j=2}^{d} \frac{\partial^{2} h}{\partial t_{j}^{2}}\right)(\delta \mathbf{t})\right||\varphi| e^{-\beta(t)} .
\end{aligned}
$$

Since the support of $h$ is compact, there exists a positive number $C_{6}$ (which depends only on $h$ and $r$ ) such that

$$
\left|2 \sqrt{-1} r \frac{\partial h}{\partial t}(\delta \mathbf{t})+\delta\left(\frac{\partial^{2} h}{\partial t^{2}}+\sum_{j=2}^{d} \frac{\partial^{2} h}{\partial t_{j}^{2}}\right)(\delta \mathbf{t})\right| \leq C_{6} .
$$


We also have

$$
\left|f_{\varphi, r, \delta}\right|=|h||\varphi| e^{-\beta(t)} \leq|\varphi| e^{-\beta(t)}
$$

Since the support of $h(\delta \mathbf{t})$ is contained in the closure of the ball $\frac{1}{\delta} \mathcal{D}$ and $\lim _{\delta \rightarrow 0} \frac{C_{4}}{\delta}=\infty$, the following holds.

For any $\varepsilon>0$, there exists a positive number $\delta(\varepsilon)$ smaller than $\varepsilon$ such that

$$
\left|\frac{(n-1)^{2} \kappa^{2}}{4}-\left\{\left(\beta^{\prime}\right)^{2}+\beta^{\prime \prime}\right\}\right|<\varepsilon
$$

on the support of $h(\delta \mathbf{t})$ for each $\delta<\delta(\varepsilon)$.

We have, for such $\delta$,

$$
\left|A_{1}(y, \mathbf{t})\right| \leq\left(\delta C_{6}+\varepsilon\right)|\varphi| e^{-\beta(t)}
$$

and

$$
\begin{aligned}
& \left\|A_{1}\right\|^{2}=\int_{Y \times \mathcal{C}}\left|A_{1}(y, \mathbf{t})\right|^{2} d \mu=\int_{\mathcal{C}} \int_{Y}\left|A_{1}(y, \mathbf{t})\right|^{2} d \mu_{Y, \mathbf{t}} d \mu_{\mathbf{t}} \\
& \leq\left(\delta C_{6}+\varepsilon\right)^{2} \int_{\mathcal{D} / \delta} \int_{Y}|\varphi|^{2} e^{-2 \beta(t)} d \mu_{Y, \mathbf{t}} d \mu_{\mathbf{t}} \\
& \quad=\left(\delta C_{6}+\varepsilon\right)^{2} \int_{\mathcal{D} / \delta} \int_{Y}|\varphi|^{2} d \mu_{Y} d \mu_{\mathbf{t}} \leq \varepsilon^{2}\left(C_{6}+1\right)^{2} \cdot \frac{1}{\delta^{d}} \operatorname{vol}(\mathcal{D}) \int_{Y}|\varphi|^{2} d \mu_{Y} .
\end{aligned}
$$

Since the support of $\varphi$ is compact, we can take positive numbers $C_{7}, C_{8}$ such that

$$
C_{7} \leq \operatorname{vol}\left(\pi^{-1}(z)\right) \leq C_{8}
$$

for all $z$ in the support of $\varphi$. Consequently, we obtain

$$
\left\|A_{1}\right\|^{2} \leq C_{8} \cdot \varepsilon^{2}\left(C_{6}+1\right)^{2} \frac{1}{\delta^{d}} \operatorname{vol}(\mathcal{D})\|\varphi\|_{B}^{2} .
$$

Similarly, we have

$$
\left|A_{2}(y, \mathbf{t})\right| \leq\left|\left(\Delta_{B}-c\right) \varphi\right| e^{-\beta(t)} .
$$

It follows that

$$
\begin{aligned}
& \left\|A_{2}\right\|^{2} \leq \int_{\mathcal{C}} \int_{Y}\left|\left(\Delta_{B}-c\right) \varphi\right|^{2} e^{-2 \beta(t)} d \mu_{Y, \mathbf{t}} d \mu_{\mathbf{t}} \\
& =\int_{\mathcal{D} / \delta} \int_{Y}\left|\left(\Delta_{B}-c\right) \varphi\right|^{2} d \mu_{Y} d \mu_{\mathbf{t}} \leq C_{8} \cdot \frac{1}{\delta^{d}} \operatorname{vol}(\mathcal{D})\left\|\left(\Delta_{B}-c\right) \varphi\right\|_{B}^{2} .
\end{aligned}
$$


Thus we obtain

$$
\left\|A_{2}\right\|^{2}<C_{8} \cdot \varepsilon^{2} \cdot \frac{1}{\delta^{d}} \operatorname{vol}(\mathcal{D})\|\varphi\|_{B}^{2} .
$$

From (1.5), (1.6) and (1.7), we have

$$
\left\|\left(\Delta-\left(\frac{(n-1)^{2} \kappa^{2}}{4}+c+r^{2}\right)\right) f_{\varphi, r, \delta}\right\|^{2}<C_{8} \varepsilon^{2}\left(C_{6}+2\right)^{2} \frac{1}{\delta^{d}} \operatorname{vol}(\mathcal{D})\|\varphi\|_{B}^{2} .
$$

On the other hand, since

$$
\begin{aligned}
& \left\|f_{\varphi, r, \delta}\right\|^{2}=\int_{Y \times \mathcal{C}}\left|f_{\varphi, r, \delta}\right|^{2} d \mu=\int_{\mathcal{C}} \int_{Y}\left|f_{\varphi, r, \delta}\right|^{2} d \mu_{Y, \mathbf{t}} d \mu_{\mathbf{t}} \\
& \geq \int_{\mathcal{D}^{\prime} / \delta} \int_{Y}\left|f_{\varphi, r, \delta}\right|^{2} d \mu_{Y, \mathbf{t}} d \mu_{\mathbf{t}} \\
& =\int_{\mathcal{D}^{\prime} / \delta} \int_{Y}|\varphi|^{2} e^{-2 \beta(t)} d \mu_{Y, \mathbf{t}} d \mu_{\mathbf{t}}=\int_{\mathcal{D}^{\prime} / \delta} \int_{Y}|\varphi|^{2} d \mu_{Y} d \mu_{\mathbf{t}}
\end{aligned}
$$

we have

$$
\left\|f_{\varphi, r, \delta}\right\|^{2} \geq C_{7} \cdot \frac{1}{\delta^{d}} \operatorname{vol}\left(\mathcal{D}^{\prime}\right)\|\varphi\|_{B}^{2}
$$

From (1.8) and (1.9), we have

$$
\begin{aligned}
\left\|\left(\Delta-\left(\frac{(n-1)^{2} \kappa^{2}}{4}+c+r^{2}\right)\right) f_{\varphi, r, \delta}\right\|^{2} & \\
& <\left(C_{6}+2\right)^{2} \cdot \frac{C_{7}}{C_{8}} \cdot \frac{\operatorname{vol}(\mathcal{D})}{\operatorname{vol}\left(\mathcal{D}^{\prime}\right)} \cdot \varepsilon^{2}\left\|f_{\varphi, r, \delta}\right\|^{2} .
\end{aligned}
$$

Let

$$
C_{5}=\left(C_{6}+2\right) \sqrt{\frac{C_{7} \operatorname{vol}(\mathcal{D})}{C_{8} \operatorname{vol}\left(\mathcal{D}^{\prime}\right)}}
$$

Then we obtain the inequality (1.4).

Proof of Theorem 1. We first prove (2). Let $\varphi_{*}$ be an eigenfunction belonging to the eigenvalue $c_{m}$. From the definition of $\bar{\Delta}_{B}$, there exists a sequence $\left\{\varphi_{i}\right\}$ in $C_{0}^{\infty}(B)$ such that $\lim _{i \rightarrow \infty} \varphi_{i}=\varphi_{*}$ and $\lim _{i \rightarrow \infty} \Delta_{B} \varphi_{i}=\bar{\Delta}_{B} \varphi_{*}$ in $L^{2}(B)$. Then, by the triangle inequality, we can find for each $\varepsilon>0$ a function $\varphi \in C_{0}^{\infty}(B)$ such that

$$
\left\|\left(\Delta_{B}-c_{m}\right) \varphi\right\|_{B}<\varepsilon\|\varphi\|_{B}
$$

Since the condition $(*)$ is satisfied, the estimate in Proposition 1.2 is valid for any positive number $\delta<\delta(\varepsilon)$. We take one of such $\delta$ and put

$$
u_{m, r, \varepsilon}=f_{\varphi, r, \delta}
$$


Choose the functions $\varphi$ so that $\left\|\varphi_{*}-\varphi\right\|_{B}$ are sufficiently small. Then the resultant family $\left\{u_{m, r, \varepsilon}\right\}_{\varepsilon>0}$ of compactly supported smooth functions on $\mathcal{E}$ satisfies the conditions $(0.3)-(0.5)$.

To prove (1), we take a constant function $\varphi \equiv 1$ and consider $f_{\varphi, r, \delta}$ in (1.3). Since $\left(\tau_{\mathbf{t}}\right)_{y} \cdot(\varphi \circ \pi)=0$ and $\Delta_{B} \varphi=0$, the estimate in Proposition 1.2 is valid for $c=0$. By repeating the same argument as above, the conclusion follows.

Remark. In the case (2), if there is another eigenfunction $\bar{\varphi}_{*}$ belonging to the eigenvalue $c_{m}$ such that $\varphi_{*}$ and $\bar{\varphi}_{*}$ are mutually orthogonal with respect to the $L^{2}$-inner product on $L^{2}(B)$, then we can construct for any $r \geq 0$ a family $\left\{\bar{u}_{m, r, \varepsilon}\right\}_{\varepsilon>0}$ of compactly supported smooth functions on $\mathcal{E}$ satisfying the similar conditions as $(0.2)-(0.5)$ and

$$
\lim _{\substack{\varepsilon \\ \varepsilon} 0}\left(u_{m, r, \varepsilon}, \bar{u}_{m, r^{\prime}, \varepsilon^{\prime}}\right)=0 .
$$

Remark. If $B$ is compact, we can take an eigenfunction of $\bar{\Delta}_{B}$ as $\varphi$ in the above proof of (2).

\section{$\S 2 . \quad$ Lower Bounds of the Essential Spectrum}

In this section we prove Theorem 2. It suffices to show the following.

There exists a finite dimensional subspace $\mathcal{V}$ of $L^{2}(V)$ such that

$$
\left(\left(\bar{\Delta}-\frac{(n-1)^{2} a^{2}}{4}\right) u, u\right)>0 \text { for all } u \in \mathcal{V}^{\perp} \cap \operatorname{dom}(\bar{\Delta})-\{0\},
$$

where $\mathcal{V}^{\perp}$ is the orthogonal complement of $\mathcal{V}$ in $L^{2}(V)$ and $\operatorname{dom}(\bar{\Delta})$ is the domain of definition of $\bar{\Delta}$.

Let

$$
\mathcal{E}_{j}^{\wedge}=\phi_{j}\left(Y_{j} \times\left(C_{3}(j), \infty\right)\right), \quad \partial_{j} W=\phi_{j}\left(Y_{j} \times\left\{C_{3}(j)\right\}\right)
$$

for each $j=1, \ldots, s$ and let

$$
W=V-\bigcup_{j=1}^{s} \mathcal{E}_{j}^{\wedge}
$$

The boundary of the compact manifold $W$ decomposes as

$$
\partial W=\bigcup_{j=1}^{s} \partial_{j} W
$$




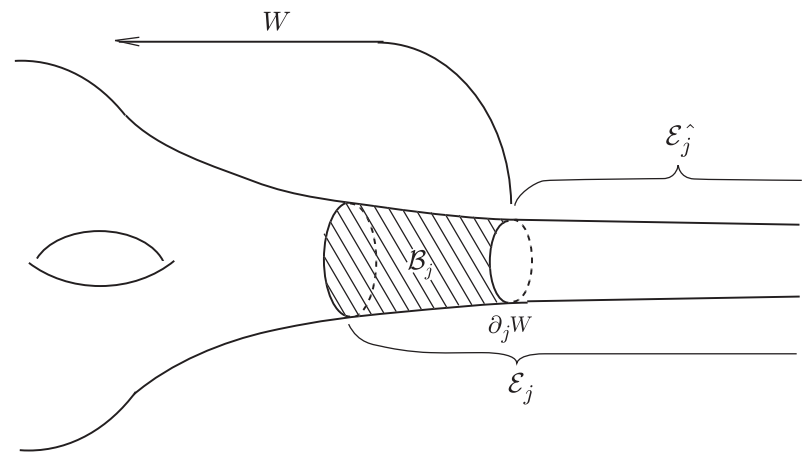

Figure 3.

We also put

$$
\mathcal{B}_{j}=\phi_{j}\left(Y_{j} \times\left(C_{2}(j), C_{3}(j)\right]\right) .
$$

Then $\mathcal{B}_{j}$ is a collar neighborhood of $\partial_{j} W$ (see Figure 3). We relate the integral in (2.1) on the noncompact manifold $V$ to some integrals on the compact manifold $W$.

For any vector field $Z$ on $V$ we denote by $|Z|(x)$ the norm of $Z_{x}$ with respect to the Riemannian metric of $V$ at the point $x \in V$. Let $L^{2}(W)$ be the space of all square integrable functions on $W$. For any $w_{1}, w_{2} \in L^{2}(W)$, let

$$
\left(w_{1}, w_{2}\right)_{W}=\int_{W} w_{1} \cdot \bar{w}_{2} d \mu
$$

be the $L^{2}$-inner product. We denote by \|\|$_{W}$ the $L^{2}$-norm on $L^{2}(W)$ :

$$
\|w\|_{W}=\sqrt{\int_{W}|w|^{2} d \mu} .
$$

Let $C^{1,2}(W)$ be the space of smooth functions $w$ on $W$ such that $|\operatorname{grad} w| \in$ $L^{2}(W)$, where grad $w$ is the gradient vector field of $w$. We denote by $\|\operatorname{grad} w\|$ the $L^{2}$-norm of $|\operatorname{grad} w|$. Let $L^{1,2}(W)$ be the completion of $C^{1,2}(V)$ with respect to the $L^{1,2}$-norm,

$$
\|w\|_{1,2}=\sqrt{\|w\|_{W}^{2}+\|\operatorname{grad} w\|_{W}^{2}} .
$$

For $u \in \operatorname{dom}(\bar{\Delta})$, we only write $\|u\|_{1,2}$ to denote the $L^{1,2}$-norm of the restriction $\left.u\right|_{W}$ of $u$ to $W:\|u\|_{1,2}=\left\|\left.u\right|_{W}\right\|_{1,2}$. 
We show the following.

There exist positive constants $C_{9}, C_{10}$ such that

$$
C_{10}\|u\|_{W}^{2}+\left(\left(\bar{\Delta}-\frac{(n-1)^{2} a^{2}}{4}\right) u, u\right) \geq C_{9}\|u\|_{1,2}^{2} \text { for all } u \in \operatorname{dom}(\bar{\Delta}) \text {. }
$$

Then the assertion (2.1) follows from (2.2): Let $\mathcal{H}$ be the image of the restriction $\left.\operatorname{map} \operatorname{dom}(\bar{\Delta}) \ni u \longmapsto u\right|_{W} \in L^{2}(W)$. It follows from Rellich-Kondrachov lemma (cf. [1, Théorème 10]) that for any positive number $\varepsilon<\sqrt{C_{9} /\left(2 C_{10}\right)}$ there exists a finite dimensional subspace $\mathcal{L}$ of $\mathcal{H}$ such that $\varepsilon\|w\|_{1,2}>\|w\|_{W}$ for any $w \neq 0$ in the orthogonal complement of $\mathcal{L}$ in $\mathcal{H}$ with respect to the inner product $(,)_{W}$ on $L^{2}(W)$. It suffices to take the image of the natural inclusion $\mathcal{L} \hookrightarrow L^{2}(W)$ as $\mathcal{V}$.

In order to prove (2.2) it suffices to show the inequality for compactly supported smooth functions $u$ on $V$. We remark that

$$
\begin{gathered}
\left(\left(\Delta-\frac{(n-1)^{2} a^{2}}{4}\right) u, u\right)=\int_{V}\left(|\operatorname{grad} u|^{2}-\frac{(n-1)^{2} a^{2}}{4}|u|^{2}\right) d \mu \\
=\int_{W}\left(|\operatorname{grad} u|^{2}-\frac{(n-1)^{2} a^{2}}{4}|u|^{2}\right) d \mu \\
+\sum_{j=1}^{s} \int_{\mathcal{E}_{j}^{\wedge}}\left(|\operatorname{grad} u|^{2}-\frac{(n-1)^{2} a^{2}}{4}|u|^{2}\right) d \mu
\end{gathered}
$$

for such $u$. Hence, we estimate the integrals in the last term of (2.3) as follows.

Lemma 2.1. For any compactly supported smooth function $u$ on $V$, we have

$$
\begin{aligned}
& \int_{\mathcal{E}_{j}^{\wedge}}\left(|\operatorname{grad} u|^{2}-\frac{(n-1)^{2} a^{2}}{4}|u|^{2}\right) d \mu \\
& \geq \beta_{j}^{\prime}\left(C_{3}(j)\right)\left(\frac{1}{C_{3}(j)-C_{2}(j)}-4 \beta_{j}^{\prime}\left(C_{3}(j)\right)\right) \int_{\mathcal{B}_{j}}|u|^{2} d \mu-\frac{1}{4} \int_{\mathcal{B}_{j}}|\operatorname{grad} u|^{2} d \mu .
\end{aligned}
$$

Proof. From now on to (2.13), we drop the index $j$ for simplicity. We write just $\mathcal{E}^{\wedge}, Y, \partial W, \mathcal{B}, \beta(t), C_{2}, C_{3}, \phi, \mathcal{K}(t)$ instead of $\mathcal{E}_{j}^{\wedge}, Y_{j}, \partial_{j} W, \mathcal{B}_{j}$, $\beta_{j}(t), C_{2}(j), C_{3}(j), \phi_{j}, \mathcal{K}_{j}(t)$, etc.

On $\mathcal{E}^{\wedge}$ we use the same local coordinate system $\left(y_{1}, \ldots, y_{n-1}, t\right)$ as in the proof of Proposition 1.1. In this case $d=1$ and we can write $u=u(y, t)$. We can decompose the gradient vector field of $u$ as

$$
\operatorname{grad} u=Z+\frac{\partial u}{\partial t} \frac{\partial}{\partial t}, \quad Z \perp \frac{\partial}{\partial t} .
$$


Then we have

$$
|\operatorname{grad} u|^{2}=|Z|^{2}+\left|\frac{\partial u}{\partial t}\right|^{2}
$$

We remark that

$$
e^{-2 \beta(t)}\left|\frac{\partial}{\partial t}\left(u e^{\beta(t)}\right)\right|^{2}=\left|\frac{\partial u}{\partial t}\right|^{2}+\left(\beta^{\prime}(t)\right)^{2}|u|^{2}+2 \beta^{\prime}(t) \operatorname{Re}\left(u \frac{\partial \bar{u}}{\partial t}\right),
$$

where $\operatorname{Re}($ ) means the real part. Since $u$ has a compact support, there exists a positive number $C_{11}=C_{11}(j)$ such that the support of $u$ is contained in $V-\phi\left(Y \times\left[C_{11}, \infty\right)\right)$. Then we have

$$
\begin{gathered}
\int_{\mathcal{E}^{\wedge}} 2 \beta^{\prime}(t) \operatorname{Re}\left(u \frac{\partial \bar{u}}{\partial t}\right) d \mu=\lim _{t \rightarrow \infty} \int_{C_{3}}^{t}\left\{\int_{Y} 2 \beta^{\prime}(t) \operatorname{Re}\left(u \frac{\partial \bar{u}}{\partial t}\right) e^{2 \beta(t)} d \mu_{Y}\right\} d t \\
=\int_{C_{3}}^{C_{11}}\left\{\int_{Y} 2 \beta^{\prime}(t) \operatorname{Re}\left(u \frac{\partial \bar{u}}{\partial t}\right) e^{2 \beta(t)} d \mu_{Y}\right\} d t,
\end{gathered}
$$

where $\mu_{Y}$ is the canonical measure of $Y$. Since

$$
\frac{\partial}{\partial t}\left[\beta^{\prime}(t)|u|^{2} e^{2 \beta(t)}\right]=\left\{2 \beta^{\prime}(t) \operatorname{Re}\left(u \frac{\partial \bar{u}}{\partial t}\right)+\beta^{\prime \prime}(t)|u|^{2}+2\left(\beta^{\prime}(t)\right)^{2}|u|^{2}\right\} e^{2 \beta(t)},
$$

we have

$$
\begin{aligned}
& \int_{\mathcal{E}^{\wedge}} 2 \beta^{\prime}(t) \operatorname{Re}\left(u \frac{\partial \bar{u}}{\partial t}\right) d \mu \\
& =\int_{C_{3}}^{C_{11}} \int_{Y}\left\{\frac{\partial}{\partial t}\left[\beta^{\prime}(t)|u|^{2} e^{2 \beta(t)}\right]-|u|^{2} \beta^{\prime \prime} e^{2 \beta(t)}-2\left(\beta^{\prime}\right)^{2}|u|^{2} e^{2 \beta(t)}\right\} d \mu_{Y} d t \\
& =-\int_{Y} \beta^{\prime}\left(C_{3}\right)|u|^{2} e^{2 \beta\left(C_{3}\right)} d \mu_{Y}-\int_{\mathcal{E}^{\wedge}}|u|^{2}\left(\beta^{\prime \prime}+2\left(\beta^{\prime}\right)^{2}\right) d \mu \\
& =-\int_{\partial W} \beta^{\prime}|u|^{2} d \nu-\int_{\mathcal{E}^{\wedge}}|u|^{2}\left(\beta^{\prime \prime}+2\left(\beta^{\prime}\right)^{2}\right) d \mu,
\end{aligned}
$$

where $\nu$ is the canonical measure on $\partial W$ induced from the Riemannian metric of $V$. From (2.5) and (2.6), we have

$$
\begin{aligned}
& \int_{\mathcal{E}^{\wedge}} e^{-2 \beta(t)}\left|\frac{\partial}{\partial t}\left(u e^{\beta}\right)\right|^{2} d \mu \\
& =\int_{\mathcal{E}^{\wedge}}\left(\left|\frac{\partial u}{\partial t}\right|^{2}-|u|^{2}\left(\beta^{\prime \prime}+\left(\beta^{\prime}\right)^{2}\right)\right) d \mu-\int_{\partial W} \beta^{\prime}|u|^{2} d \nu
\end{aligned}
$$


From (2.4), (2.7), we have

$$
\begin{aligned}
& \int_{\mathcal{E}^{\wedge}}\left(|\operatorname{grad} u|^{2}-|u|^{2}\left(\beta^{\prime \prime}+\left(\beta^{\prime}\right)^{2}\right)\right) d \mu \\
& =\int_{\mathcal{E}^{\wedge}}\left\{|Z|^{2}+e^{-2 \beta(t)}\left|\frac{\partial}{\partial t}\left(u e^{\beta(t)}\right)\right|^{2}\right\} d \mu+\int_{\partial W} \beta^{\prime}|u|^{2} d \nu \\
& \geq \int_{\partial W} \beta^{\prime}|u|^{2} d \nu
\end{aligned}
$$

Let us estimate the integral $\int_{\partial W} \beta^{\prime}|u|^{2} d \nu$ from below. Put $\psi(t)=(t-$ $\left.C_{2}\right) /\left(C_{3}-C_{2}\right)$. Then we have

$$
\begin{aligned}
& \left|u\left(y, C_{3}\right)\right|^{2} e^{2 \beta\left(C_{3}\right)}=e^{2 \beta\left(C_{3}\right)} \int_{C_{2}}^{C_{3}} \frac{\partial}{\partial t}\left\{\psi(t)|u|^{2}\right\} d t \\
& =e^{2 \beta\left(C_{3}\right)} \int_{C_{2}}^{C_{3}}\left\{\frac{|u|^{2}}{C_{3}-C_{2}}+2 \psi \operatorname{Re}\left(u \frac{\partial \bar{u}}{\partial t}\right)\right\} d t .
\end{aligned}
$$

We remark that $\beta\left(C_{3}\right) \leq \beta(t)$ on $\left[C_{2}, C_{3}\right]$. It follows that

$$
\left|u\left(y, C_{3}\right)\right|^{2} e^{2 \beta\left(C_{3}\right)} \leq \int_{C_{2}}^{C_{3}} \frac{|u|^{2} e^{2 \beta(t)}}{C_{3}-C_{2}} d t+2 \int_{C_{2}}^{C_{3}}\left|u \frac{\partial \bar{u}}{\partial t}\right| e^{2 \beta(t)} d t .
$$

Since

$$
\left|u \frac{\partial \bar{u}}{\partial t}\right| e^{2 \beta(t)}=|u| \cdot 2 \sqrt{-\beta^{\prime}\left(C_{3}\right)} e^{\beta(t)} \times\left|\frac{\partial \bar{u}}{\partial t}\right| \frac{1}{2 \sqrt{-\beta^{\prime}\left(C_{3}\right)}} e^{\beta(t)},
$$

we have, from the arithmetic geometric mean inequality,

$$
\begin{aligned}
& 2 \int_{C_{2}}^{C_{3}}\left|u \frac{\partial \bar{u}}{\partial t}\right| e^{2 \beta(t)} d t \\
& \leq 2 \sqrt{\int_{C_{2}}^{C_{3}}(-4) \beta^{\prime}\left(C_{3}\right)|u|^{2} e^{2 \beta(t)} d t} \sqrt{\int_{C_{2}}^{C_{3}} \frac{1}{-4 \beta^{\prime}\left(C_{3}\right)}\left|\frac{\partial \bar{u}}{\partial t}\right|^{2} e^{2 \beta(t)} d t} \\
& \leq-4 \beta^{\prime}\left(C_{3}\right) \int_{C_{2}}^{C_{3}}|u|^{2} e^{2 \beta(t)} d t-\frac{1}{4 \beta^{\prime}\left(C_{3}\right)} \int_{C_{2}}^{C_{3}}\left|\frac{\partial \bar{u}}{\partial t}\right|^{2} e^{2 \beta(t)} d t .
\end{aligned}
$$

We obtain

$$
\begin{aligned}
& -\beta^{\prime}\left(C_{3}\right)\left|u\left(y, C_{3}\right)\right|^{2} e^{2 \beta\left(C_{3}\right)} \\
& \leq-\beta^{\prime}\left(C_{3}\right)\left(\frac{1}{C_{3}-C_{2}}-4 \beta^{\prime}\left(C_{3}\right)\right) \int_{C_{2}}^{C_{3}}|u|^{2} e^{2 \beta(t)} d t \\
& +\frac{1}{4} \int_{C_{2}}^{C_{3}}|\operatorname{grad} u|^{2} e^{2 \beta(t)} d t .
\end{aligned}
$$


Hence

$$
\begin{aligned}
& -\int_{\partial W} \beta^{\prime}|u|^{2} d \nu=-\beta^{\prime}\left(C_{3}\right) \int_{Y}|u|^{2} e^{2 \beta\left(C_{3}\right)} d \mu_{Y} \\
& \leq-\beta^{\prime}\left(C_{3}\right)\left(\frac{1}{C_{3}-C_{2}}-4 \beta^{\prime}\left(C_{3}\right)\right) \int_{Y} \int_{C_{2}}^{C_{3}}|u|^{2} e^{2 \beta(t)} d t d \mu_{Y} \\
& \quad+\frac{1}{4} \int_{Y} \int_{C_{2}}^{C_{3}}|\operatorname{grad} u|^{2} e^{2 \beta(t)} d t d \mu_{Y} \\
& =-\beta^{\prime}\left(C_{3}\right)\left(\frac{1}{C_{3}-C_{2}}-4 \beta^{\prime}\left(C_{3}\right)\right) \int_{\mathcal{B}}|u|^{2} d \mu+\frac{1}{4} \int_{\mathcal{B}}|\operatorname{grad} u|^{2} d \mu,
\end{aligned}
$$

and

$$
\begin{aligned}
& \int_{\partial W} \beta^{\prime}|u|^{2} d \nu \\
& \geq \beta^{\prime}\left(C_{3}\right)\left(\frac{1}{C_{3}-C_{2}}-4 \beta^{\prime}\left(C_{3}\right)\right) \int_{\mathcal{B}}|u|^{2} d \mu-\frac{1}{4} \int_{\mathcal{B}}|\operatorname{grad} u|^{2} d \mu .
\end{aligned}
$$

Combining (2.8) with (2.11), we obtain

$$
\begin{aligned}
& \int_{\mathcal{E}^{\wedge}}\left(|\operatorname{grad} u|^{2}-|u|^{2}\left(\beta^{\prime \prime}+\left(\beta^{\prime}\right)^{2}\right) d \mu\right. \\
& \geq \beta^{\prime}\left(C_{3}\right)\left(\frac{1}{C_{3}-C_{2}}-4 \beta^{\prime}\left(C_{3}\right)\right) \int_{\mathcal{B}}|u|^{2} d \mu-\frac{1}{4} \int_{\mathcal{B}}|\operatorname{grad} u|^{2} d \mu .
\end{aligned}
$$

From Proposition 1.1 and the assumption, we have

$$
\beta^{\prime \prime}+\left(\beta^{\prime}\right)^{2}=\frac{(n-1)^{2}}{4}\left\{\mathcal{K}(t)-\frac{2}{n-1} \mathcal{K}^{\prime}(t)\right\} \geq \frac{(n-1)^{2} a^{2}}{4}
$$

The desired inequality now follows from (2.12) and (2.13).

Since $\beta_{j}^{\prime}\left(C_{3}(j)\right) \leq 0$ and $\bigcup_{j=1}^{s} \mathcal{B}_{j} \subset W$, we have, from (2.3) and Lemma 
2.1

$$
\begin{gathered}
\left(\left(\Delta-\frac{(n-1)^{2} a^{2}}{4}\right) u, u\right)-\int_{W}\left(|\operatorname{grad} u|^{2}-\frac{(n-1)^{2} a^{2}}{4}|u|^{2}\right) d \mu \\
\geq \sum_{j=1}^{s} \beta_{j}^{\prime}\left(C_{3}(j)\right)\left(\frac{1}{C_{3}(j)-C_{2}(j)}-4 \beta_{j}^{\prime}\left(C_{3}(j)\right)\right) \int_{\mathcal{B}_{j}}|u|^{2} d \mu \\
-\frac{1}{4} \sum_{j=1}^{s} \int_{\mathcal{B}_{j}}|\operatorname{grad} u|^{2} d \mu \\
\geq\left\{\sum_{j=1}^{s} \beta_{j}^{\prime}\left(C_{3}(j)\right)\left(\frac{1}{C_{3}(j)-C_{2}(j)}-4 \beta_{j}^{\prime}\left(C_{3}(j)\right)\right)\right\} \int_{W}|u|^{2} d \mu \\
-\frac{1}{4} \int_{W}|\operatorname{grad} u|^{2} d \mu .
\end{gathered}
$$

Therefore,

$$
\begin{aligned}
& \left(\left(\Delta-\frac{(n-1)^{2} a^{2}}{4}\right) u, u\right) \\
& +\left\{\frac{(n-1)^{2} a^{2}}{4}+\sum_{j=1}^{s}\left(-\beta_{j}^{\prime}\left(C_{3}(j)\right)\left(\frac{1}{C_{3}(j)-C_{2}(j)}-4 \beta_{j}^{\prime}\left(C_{3}(j)\right)\right)\right)+\frac{3}{4}\right\} \\
& \times \int_{W}|u|^{2} d \mu \geq \frac{3}{4} \int_{W}\left\{|u|^{2}+|\operatorname{grad} u|^{2}\right\} d \mu .
\end{aligned}
$$

Let $C_{9}=3 / 4$ and

$C_{10}=\frac{(n-1)^{2} a^{2}}{4}+\frac{3}{4}+\max _{1 \leq j \leq s}\left\{-\beta_{j}^{\prime}\left(C_{3}(j)\right)\left(\frac{1}{C_{3}(j)-C_{2}(j)}-4 \beta_{j}^{\prime}\left(C_{3}(j)\right)\right)\right\}$.

This proves the inequality (2.2), and Theorem 2 now follows.

Proof of Lemma 1. For simplicity, we omit the symbol $d \mu_{N}$. Let $v$ be a non-negative compactly supported smooth function on $N$. Since

$$
\operatorname{div} v Z=\langle\operatorname{grad} v, Z\rangle+v \cdot \operatorname{div} Z
$$

we have

$$
0=\int_{N} \operatorname{div} v Z=\int_{N}\langle\operatorname{grad} v, Z\rangle+\int_{N} v \cdot \operatorname{div} Z \geq \int_{N}\langle\operatorname{grad} v, Z\rangle+\varepsilon \int_{N} v .
$$

Hence

$$
\varepsilon \int_{N} v \leq-\int_{N}\langle\operatorname{grad} v, Z\rangle \leq \int_{N}|\operatorname{grad} v| \cdot|Z|
$$


and

$$
\int_{N} v \leq \frac{C}{\varepsilon} \int_{N}|\operatorname{grad} v| .
$$

For any compactly supported smooth function $u$ on $N$, let $v=u^{2}$. Since $\operatorname{grad} u^{2}=2 u \cdot \operatorname{grad} u$, we have

$$
\begin{aligned}
\int_{N} u^{2} & \leq \frac{C}{\varepsilon} \int_{N}\left|\operatorname{grad} u^{2}\right| \leq \frac{2 C}{\varepsilon} \int_{N}|u| \cdot|\operatorname{grad} u| \\
& \leq \frac{2 C}{\varepsilon}\left(\int_{N}|u|^{2}\right)^{1 / 2}\left(\int_{N}|\operatorname{grad} u|^{2}\right)^{1 / 2}
\end{aligned}
$$

and

$$
\left(\int_{N}|u|^{2}\right)^{1 / 2} \leq \frac{2 C}{\varepsilon}\left(\int_{N}|\operatorname{grad} u|^{2}\right)^{1 / 2} .
$$

Therefore, we obtain

$$
\left(\frac{\varepsilon}{2 C}\right)^{2} \leq \frac{\int_{N}|\operatorname{grad} u|^{2}}{\int_{N}|u|^{2}}
$$

\section{$\S 3 . \quad$ The Ends of Higher $\mathbb{Q}$-Rank Locally Symmetric Spaces}

In this section we explain the case of higher $\mathbb{Q}$-rank locally symmetric spaces of finite volume and state a similar result to Theorem 3. The situation is slightly different from the one in Theorem 1 . There are fiber bundles $\pi$ : $Y \longrightarrow B$, cones $\mathcal{C}$, and Riemannian metrics on $Y \times \mathcal{C}$ satisfying the conditions $(\star \star),(*)$, and $(* *)$. However, $Y \times \mathcal{C}$ is not entirely contained in $V$ when $B$ is not compact. Instead, there exist an exhaustion $\mathcal{W}_{1} \subset \mathcal{W}_{2} \subset \cdots \subset \mathcal{W}_{i} \subset \cdots$ of $B$ by relatively compact open subsets, a corresponding nested sequence $\mathcal{C}_{1} \supset \mathcal{C}_{2} \supset$ $\cdots \supset \mathcal{C}_{i} \supset \cdots$ of cones in $\mathcal{C}$, and an embedding $\bigcup_{i=1}^{\infty}\left(\pi^{-1}\left(\mathcal{W}_{i}\right) \times \mathcal{C}_{i}\right) \longrightarrow V$. In the sequel, for any algebraic group $\mathbf{H}$ defined over $\mathbb{Q}$, we denote by $\mathbf{H}(\mathbb{R})$, $\mathbf{H}(\mathbb{Q}), \mathbf{H}(\mathbb{Z})$ the group of real, rational, integral points of $\mathbf{H}$, respectively.

Let $G$ be a connected semisimple Lie group having finite center and no compact factors. Let $K$ be a maximal compact subgroup of $G$ and $X=G / K$ the associated symmetric space of noncompact type with the canonical left invariant metric $\widetilde{g}$. Let $\Gamma$ be an irreducible non-uniform lattice of $X$. Suppose that the rank of $X$ is at least 2 and $G$ has trivial center. Then, by the arithmeticity theorem of G.A. Margulis (see [30]), there exist a semisimple linear algebraic group $\mathbf{G}$ defined over $\mathbb{Q}$ and an isomorphism (of Lie groups) from $G$ to the identity component of $\mathbf{G}(\mathbb{R})$ such that the image of $\Gamma$ is contained in $\mathbf{G}(\mathbb{Q})$ and is commensurable with $\mathbf{G}(\mathbb{Z})$. We suppose that $\Gamma$ is torsion-free and that the $\mathbb{Q}$-rank $r_{\mathbb{Q}}(\mathbf{G})$ of $\mathbf{G}$ is at least 2 . Then there is a compactification 
$\bar{V}$ of the quotient manifold $V=\Gamma \backslash X$ constructed by A. Borel and J.-P. Serre such that $\bar{V}$ is a manifold with corners and its boundary $\partial \bar{V}$ is connected ([7, Corollary 8.6.2]). Hence $V$ has only one end. Let $x_{0}$ be the coset in $X$ of the identity element, $\Pi: X \longrightarrow V$ the natural projection, and $g$ the metric on $V$ such that $\Pi^{*} g=\widetilde{g}$.

We first recall some facts about parabolic subgroups of $\mathbf{G}$ (see [3], [29] for more details). Let $\mathbf{S}$ be a maximal $\mathbb{Q}$-split torus of $\mathbf{G}$ and $\Phi(\mathbf{G}, \mathbf{S})$ the system of rational roots of $\mathbf{G}$ with respect to $\mathbf{S}$. Let $\mathbb{R} \mathfrak{g}, \mathfrak{g}$, and $\mathfrak{k}$ be the Lie algebras of $G, \mathbf{G}$, and $K$, respectively. We denote by exp the exponential mapping from $\mathbb{R} \mathfrak{g}$ to $G$. Let $\mathbb{R} \mathfrak{g}=\mathfrak{k}+\mathfrak{p}$ be the Cartan decomposition, where $\mathfrak{p}$ is the orthogonal complement of $\mathfrak{k}$ in $\mathbb{R} \mathfrak{g}$ with respect to the Killing form of $\mathbb{R} \mathfrak{g}$. We denote by $\langle$,$\rangle the inner product on \mathfrak{p}$ induced from the Riemannian metric on the tangent space $T_{x_{0}}(X)$ of $X$ at $x_{0}$. Then $\langle$,$\rangle coincides with the restriction$ of the inner product $\langle\langle\rangle$,$\rangle on \mathbb{R} \mathfrak{g}$ obtained from the Killing form of $\mathbb{R} \mathfrak{g}$ and the Cartan involution of $\mathbb{R} \mathfrak{g}$. Let $A$ be the identity component of $\mathbf{S}(\mathbb{R})$ and $\mathfrak{a}$ its Lie algebra. By considering the restriction to $\mathfrak{a}$ of the differential of each rational root $\mathbf{S} \longrightarrow \mathbb{C}^{*}$, we can regard $\Phi(\mathbf{G}, \mathbf{S})$ as the system $\Sigma$ of roots of the pair $(\mathbb{R} \mathfrak{g}, \mathfrak{a})$. For each root $\theta \in \Sigma$, let $H_{\theta}$ be the unique element of $\mathfrak{a}$ such that $\theta(H)=\left\langle H_{\theta}, H\right\rangle$ for all $H \in \mathfrak{a}$. We introduce a lexicographic order into $\Phi(\mathbf{G}, \mathbf{S})$, and denote by $\Phi^{+}(\mathbf{G}, \mathbf{S})$ the set of positive rational roots with respect to this order. We introduce the corresponding order into $\Sigma$, and denote by $\Sigma^{+}$ the set of positive roots corresponding to $\Phi^{+}(\mathbf{G}, \mathbf{S})$. We put

$$
\mathfrak{a}^{+}=\left\{H \in \mathfrak{a} \mid \theta(H) \geq 0 \text { for all } \theta \in \Sigma^{+}\right\}
$$

and

$$
\rho=\frac{1}{2} \sum_{\theta \in \Sigma^{+}} H_{\theta},
$$

where in the sum every root occurs a number of times equal to its multiplicity. Let

$$
\mathfrak{g}=\mathfrak{g}_{0}+\coprod_{\chi \in \Phi(\mathbf{G}, \mathbf{S})} \mathfrak{g}_{\chi}
$$

be the root space decomposition of $\mathfrak{g}$. Let $\mathfrak{u}$ be the subalgebra of $\mathfrak{g}$ defined by

$$
\mathfrak{u}=\coprod_{\chi \in \Phi^{+}(\mathbf{G}, \mathbf{S})} \mathfrak{g}_{\chi}
$$

and $\mathbf{U}$ the analytic subgroup with Lie algebra $\mathfrak{u}$. Let $\Upsilon \subset \Sigma^{+}$be the set of positive simple roots of $(\mathbb{R} \mathfrak{g}, \mathfrak{a})$ and $\Upsilon(\mathbf{G}) \subset \Phi^{+}(\mathbf{G}, \mathbf{S})$ the corresponding set of 
positive simple roots. For any subset $I \subset \Upsilon$, we take the corresponding subset $I(\mathbf{G}) \subset \Upsilon(\mathbf{G})$ and define a subgroup $\mathbf{S}_{I}$ of $\mathbf{S}$ by

$$
\mathbf{S}_{I}=\left(\bigcap_{\alpha \in I(\mathbf{G})} \operatorname{ker} \alpha\right)^{0}
$$

where ()$^{0}$ means the identity component with respect to the Zariski topology. The group $\mathbf{P}_{I}=Z\left(\mathbf{S}_{I}\right) \mathbf{U}$, where $Z\left(\mathbf{S}_{I}\right)$ is the centralizer of $\mathbf{S}_{I}$ in $\mathbf{G}$, is a rational parabolic subgroup of $\mathbf{G}$ containing $\mathbf{S}$. These are called the standard rational parabolic subgroups of $\mathbf{G}$. The unipotent radical of $\mathbf{P}_{I}$, that is, the greatest connected unipotent normal subgroup of $\mathbf{P}_{I}$, is the analytic subgroup $\mathbf{U}_{I}$ with Lie algebra

$$
\mathfrak{u}_{I}=\coprod^{\prime} \mathfrak{g}_{\chi}
$$

where the sum is over all the positive rational roots which are not linear combinations of elements of $I$. If $I$ and $J$ are two subsets of $\Upsilon$ such that $I \subset J$, then $\mathbf{P}_{I} \subset \mathbf{P}_{J}$. The rational parabolic subgroup $\mathbf{P}=\mathbf{P}_{\emptyset}=Z(\mathbf{S}) \mathbf{U}$ is a minimal one containing $\mathbf{S}$, and $\mathbf{P}_{\Upsilon}=\mathbf{G}$. Each proper rational parabolic subgroup $\mathbf{Q}$ of $\mathbf{G}$ is conjugate by some element of $\mathbf{G}(\mathbb{Q})$ to one and only one of the $\mathbf{P}_{I}$ with $I \neq \Upsilon$; $\mathbf{Q}$ is also expressed as $k \mathbf{P}_{I} k^{-1}$ for some $k \in K$. For each $I$, the $\Gamma$-conjugacy classes of $\mathbf{P}_{I}$ are known to be finite ([4]).

Each group $\mathbf{P}_{I}$ decomposes further. We put

$$
\mathbf{M}_{I}=\bigcap_{\chi \in \mathrm{X}\left(Z\left(\mathbf{S}_{I}\right)\right)} \operatorname{ker}\left(\chi^{2}\right),
$$

where $\mathrm{X}\left(Z\left(\mathbf{S}_{I}\right)\right)$ is the group of rational characters of $Z\left(\mathbf{S}_{I}\right)$. Then $Z\left(\mathbf{S}_{I}\right)(\mathbb{R})$ is the direct product $\mathbf{M}_{I}(\mathbb{R}) \times \mathbf{S}_{I}(\mathbb{R})$. Let $A_{I}$ be the identity component of $\mathbf{S}_{I}(\mathbb{R})$ and let $M_{I}=\mathbf{M}_{I}(\mathbb{R}), U_{I}=\mathbf{U}_{I}(\mathbb{R})$. Then we have the Langlands decomposition

$$
\mathbf{P}_{I}(\mathbb{R})=U_{I} A_{I} M_{I} .
$$

The boundary $\partial \bar{V}$ of the Borel-Serre compactification $\bar{V}$ is a disjoint union of faces $e^{\prime}(\mathbf{Q})$ corresponding to the $\Gamma$-conjugacy classes of proper rational parabolic subgroups of $\mathbf{G}$. Suppose that the $\Gamma$-conjugacy classes of $\mathbf{P}_{I}(I \neq \Upsilon)$ are represented by $\mathbf{P}_{I, j}=k_{I, j} \mathbf{P}_{I} k_{I, j}^{-1}, j \in\{1, \ldots, s(I)\}$, where $k_{I, j} \in K$ for each $j$ and $k_{I, 1}=e$. In particular, $\mathbf{P}_{I, 1}=\mathbf{P}_{I}$. We briefly describe the faces $e^{\prime}\left(\mathbf{P}_{I, j}\right)$ (see [7], [31] for more details).

Let

$$
U_{I, j}=k_{I, j} U_{I} k_{I, j}^{-1}, \quad A_{I, j}=k_{I, j} A_{I} k_{I, j}^{-1}, \quad \text { and } M_{I, j}=k_{I, j} M_{I} k_{I, j}^{-1} .
$$




$$
\begin{aligned}
X \supset e\left(\mathbf{P}_{I, j}\right) & \stackrel{\tilde{\pi}_{I, j}}{\longrightarrow} X_{I, j} \\
\Pi\left|\varpi_{I, j}\right| \Gamma_{I, j} \backslash & \Pi_{I, j} \mid \Gamma_{M_{I, j}} \backslash \\
V \not \supset e^{\prime}\left(\mathbf{P}_{I, j}\right) & \underset{\pi_{I, j}}{\longrightarrow} V_{I, j}
\end{aligned}
$$

Figure 4 .

Let

$$
e\left(\mathbf{P}_{I, j}\right)=U_{I, j} M_{I, j} \cdot x_{0}=k_{I, j} U_{I} M_{I} \cdot x_{0}, \quad X_{I, j}=M_{I, j} \cdot x_{0}=k_{I, j} M_{I} \cdot x_{0} .
$$

Then $X_{I, j}$ is a product of a symmetric space of noncompact type with a possible Euclidean space, and $e\left(\mathbf{P}_{I, j}\right)$ is diffeomorphic to $U_{I, j} \times X_{I, j}$. In particular, we have a fiber bundle

$U_{I, j} \longrightarrow e\left(\mathbf{P}_{I, j}\right) \stackrel{\tilde{\pi}_{I, j}}{\longrightarrow} X_{I, j}, \quad \tilde{\pi}_{I, j}\left(k_{I, j} u m \cdot x_{0}\right)=k_{I, j} m \cdot x_{0}$ for $u \in U_{I}, m \in M_{I}$.

Let

$$
\Gamma_{I, j}=\Gamma \cap \mathbf{P}_{I, j}, \quad \Gamma_{M_{I, j}}=\left(\Gamma_{I, j} U_{I, j}\right) \cap M_{I, j}, \quad \text { and } \Gamma_{U_{I, j}}=\Gamma \cap U_{I, j} .
$$

Then $\Gamma_{I, j}$ is the semi-direct product $\Gamma_{U_{I, j}} \rtimes \Gamma_{M_{I, j}}$ and acts on $e\left(\mathbf{P}_{I, j}\right)$. Let $V_{I, j}=\Gamma_{M_{I, j}} \backslash X_{I, j}$ and $\Pi_{I, j}: X_{I, j} \longrightarrow V_{I, j}$ the natural projection. The face $e^{\prime}\left(\mathbf{P}_{I, j}\right)$ is defined by

$$
e^{\prime}\left(\mathbf{P}_{I, j}\right)=\Gamma_{I, j} \backslash e\left(\mathbf{P}_{I, j}\right) .
$$

Let $\pi_{I, j}$ be the unique map from $e^{\prime}\left(\mathbf{P}_{I, j}\right)$ to $V_{I, j}$ such that the diagram in Figure 4 is commutative. In the diagram, $\varpi_{I, j}: e\left(\mathbf{P}_{I, j}\right) \longrightarrow e^{\prime}\left(\mathbf{P}_{I, j}\right)$ is the natural projection. Then $e^{\prime}\left(\mathbf{P}_{I, j}\right)$ is a fiber bundle over $V_{I, j}$ :

$$
F_{I, j}=\Gamma_{U_{I, j}} \backslash U_{I, j} \longrightarrow e^{\prime}\left(\mathbf{P}_{I, j}\right) \stackrel{\pi_{I, j}}{\longrightarrow} V_{I, j} .
$$

The fiber $F_{I, j}$ of the fiber bundle (3.2) is a compact nilmanifold and the base space $V_{I, j}$ is a locally symmetric space of finite volume, which is compact if and only if $I=\emptyset$.

In the compactification $\bar{V}$ each face $e^{\prime}\left(\mathbf{P}_{I, j}\right)$ is located on the ideal boundary $\partial \bar{V}$. For $t>0$, we put

$A_{I}(t)=\left\{a \in A_{I} \mid \chi(a)>t\right.$ for all $\left.\chi \in \Upsilon(\mathbf{G})-I(\mathbf{G})\right\}, \quad A_{I, j}(t)=k_{I, j} A_{I}(t) k_{I, j}^{-1}$.

We just write $A(t)$ instead of $A_{\emptyset}(t)$. 
Proposition 3.1 ([7, 10.3], $[31,(1.5)])$. Let $\mathcal{W}$ be a relatively compact open subset of $V_{I, j}$. Then there exists $t^{*}=t^{*}(\mathcal{W})>0$ such that for any $t \geq t^{*}$ the set $E_{I, j, \mathcal{W}, t}=U_{I, j} A_{I, j}(t) \cdot \Pi_{I, j}^{-1}(\mathcal{W})$ is $\Gamma_{I, j}$-invariant and

$$
\left\{g \in \Gamma \mid g E_{I, j, \mathcal{W}, t} \cap E_{I, j, \mathcal{W}, t} \neq \emptyset\right\}=\Gamma_{I, j} .
$$

The equivalence relation defined on $E_{I, j, \mathcal{W}, t}$ by $\Gamma$ is the same as the one defined by $\Gamma_{I, j}$.

Let $\mathcal{W}$ be a relatively compact subset of $V_{I, j}$. We choose a positive number $t^{* *}$ such that $t^{* *} \geq t^{*}(\mathcal{W})$ and put

$$
E_{I, j, \mathcal{W}}=E_{I, j, \mathcal{W}, t^{* *}}=U_{I, j} A_{I, j}\left(t^{* *}\right) \cdot \Pi_{I, j}^{-1}(\mathcal{W}), \quad \mathcal{E}_{I, j, \mathcal{W}}=\Pi\left(E_{I, j, \mathcal{W}}\right) .
$$

Let

$$
Y_{I, j, \mathcal{W}}=\pi_{I, j}^{-1}(\mathcal{W}) \subset e^{\prime}\left(\mathbf{P}_{I, j}\right) .
$$

Then the open submanifold $\mathcal{E}_{I, j, \mathcal{W}}$ of $V$ is diffeomorphic to the product $Y_{I, j, \mathcal{W}} \times$ $\left(A_{I, j}\left(t^{* *}\right) \cdot x_{0}\right)$. Let

$$
\mathfrak{a}_{I}=\{H \in \mathfrak{a} \mid \theta(H)=0 \quad \text { for all } \theta \in I\}
$$

be the Lie algebra of $A_{I}$. We denote by $\rho_{I}$ the orthogonal projection of $\rho$ on $\mathfrak{a}_{I}^{+}:=\mathfrak{a}_{I} \cap \mathfrak{a}^{+}$and put $\rho_{I, j}=\operatorname{Ad}\left(k_{I, j}\right) \rho_{I}$. We define a geodesic ray $\gamma_{I, j}$ : $[0, \infty) \longrightarrow X$ by

$$
\gamma_{I, j}(t)=\exp \left(t \rho_{I, j} /\left|\rho_{I, j}\right|\right) \cdot x_{0} .
$$

Then, for sufficiently large $t^{* * *}>0$, the restriction of the geodesic $\Pi \circ \gamma_{I, j}$ to the interval $\left[t^{* * *}, \infty\right)$ is contained in $\mathcal{E}_{I, j, \mathcal{W}}$. If we regard $A_{I, j}\left(t^{* *}\right) \cdot x_{0}$ as an open cone $\mathcal{C}$ in $\mathbb{R}^{d}$ with

$$
d=\# \Upsilon-\# I=r_{\mathbb{Q}}(\mathbf{G})-\# I,
$$

the parabolic $\mathbb{Q}$-rank of $\mathbf{P}_{I, j}$, and regard $\left.\gamma_{I, j}\right|_{\left[t^{* * *}, \infty\right)}$ as a ray $l$ in $\mathcal{C}$, we obtain an embedding $\phi_{I, j, \mathcal{W}}: Y_{I, j, \mathcal{W}} \times \mathcal{C} \longrightarrow V$ as in the introduction. In the case where $V_{I, j}$ is not compact, let $\mathcal{W}_{1} \subset \mathcal{W}_{2} \subset \cdots \subset \mathcal{W}_{i} \subset \cdots$ be an exhaustion of $V_{I, j}$ by relatively compact subsets. Then, from the above construction, we obtain an embedding $\bigcup_{i=1}^{\infty} \mathcal{E}_{I, j, \mathcal{W}_{i}} \longrightarrow V$.

Lemma 3.1. The metric on $Y_{I, j, \mathcal{W}} \times \mathcal{C}$ induced by $\phi_{I, j, \mathcal{W}}$ is of the form $g_{\mathbf{t}}+d \mathbf{t}^{2}$ and the conditions $(\star \star),(*),(* *)$ in the introduction are satisfied. In particular,

$$
\alpha(t)=\sqrt{g_{\mathbf{t}}} / \sqrt{g_{0}}=e^{-2\left|\rho_{I}\right| t} .
$$


Proof. We only prove the case $j=1$, since other cases are similar. In this case, $U_{I, 1}=U_{I}, A_{I, 1}=A_{I}$, and $M_{I, 1}=M_{I}$. We write $\phi$ instead of $\phi_{I, 1, \mathcal{W}}$. Let $\left(t, t_{2}, \ldots, t_{d}\right)$ be a Cartesian coordinate system of $\mathbb{R}^{d}$ such that the origin $\mathbf{0}$ is $l(0)=\gamma_{I, 1}\left(t^{* * *}\right)$ and that the positive part of the $t$-axis corresponds to the ray $l$. For each $\mathbf{t}=\left(t, t_{2}, \ldots, t_{d}\right) \in \mathbb{R}^{d}$, let $a(\mathbf{t})=a\left(t, t_{2}, \ldots, t_{d}\right)$ be the unique element of $A_{I}$ such that $\mathbf{t}=a(\mathbf{t}) \cdot x_{0}$ and let $H(\mathbf{t})=H\left(t, t_{2}, \ldots, t_{d}\right)$ be the unique element of $\mathfrak{a}_{I}$ such that $\exp (H(\mathbf{t}))=a(\mathbf{t})$. We identify $e\left(\mathbf{P}_{I, 1}\right)$ with $U_{I} \times X_{I, 1}$ and define a diffeomorphism $\Phi$ from $e\left(\mathbf{P}_{I, 1}\right) \times \mathbb{R}^{d}=U_{I} \times X_{I, 1} \times \mathbb{R}^{d}$ to $V$ by

$$
\Phi\left(u m \cdot x_{0}, \mathbf{t}\right)=u a(\mathbf{t}) m \cdot x_{0} \quad \text { for } u \in U_{I}, m \in M_{I} .
$$

Let

$$
\widetilde{\mathcal{W}}=\Pi_{I, 1}^{-1}(\mathcal{W}) \subset X_{I, 1}, \quad \widetilde{Y}_{I, 1, \mathcal{W}}=U_{I} \cdot \widetilde{\mathcal{W}} \subset e\left(\mathbf{P}_{I, 1}\right) .
$$

Then $\widetilde{Y}_{I, 1, \mathcal{W}}$ is diffeomorphic to $U_{I} \times \widetilde{\mathcal{W}}$ and the map $\widetilde{\phi}: \widetilde{Y}_{I, 1, \mathcal{W}} \times \mathcal{C} \longrightarrow X$ obtained by restricting $\Phi$ on $\widetilde{Y}_{I, 1, \mathcal{W}} \times \mathcal{C}$ is an embedding. If we write $\varpi$ instead of $\varpi_{I, 1}$, we have $\phi \circ\left(\varpi \times i d_{\mathcal{C}}\right)=\Pi \circ \widetilde{\phi}$. Thus, in order to study the metric $\phi^{*} g$ it suffices to study $\widetilde{\phi}^{*} \widetilde{g}$.

Let $\Upsilon_{I}(\mathbf{G})$ be the set of maps obtained by restricting elements of $\Upsilon(\mathbf{G})-$ $I(\mathbf{G})$ to $\mathbf{S}_{I}$. For each $\alpha \in \Upsilon_{I}(\mathbf{G})$, let

$$
\mathfrak{u}_{\alpha}=\left\{X \in \mathbb{R} \mathfrak{g} \mid(\operatorname{Ad} a) X=\alpha(a) X \quad \text { for all } a \in A_{I}\right\} .
$$

Then we also have

$$
\mathfrak{u}_{I}=\coprod_{\alpha \in \Upsilon_{I}(\mathbf{G})} \mathfrak{u}_{\alpha} .
$$

The spaces $\mathfrak{u}_{\alpha}\left(\alpha \in \Upsilon_{I}(\mathbf{G})\right)$ are mutually orthogonal with respect to the inner product $\langle\langle\rangle$,$\rangle . For each \alpha \in \Upsilon_{I}(\mathbf{G})$, let $h_{\alpha}$ be the left invariant tensor field of type $(0,2)$ on $U_{I}$ which is zero on $\mathfrak{u}_{\beta}$ for $\beta \neq \alpha$, and equal to $\langle\langle\rangle$,$\rangle on \mathfrak{u}_{\alpha}$. Then

$$
d u^{2}:=\sum_{\alpha \in \Upsilon_{I}(\mathbf{G})} h_{\alpha}
$$

is a left invariant metric on $U_{I}$. For $m \in M_{I}$, we denote by Int $m$ the inner automorphism of $U_{I}$ given by

$$
\text { (Int } m)(u)=m u m^{-1} \text { for } u \in U_{I} .
$$

Let $\widetilde{g}_{X_{I, 1}}$ be the metric on $X_{I, 1}$ induced from $X$ by the natural inclusion. Since the tangent space of $\widetilde{Y}_{I, 1, \mathcal{W}} \times \mathcal{C}$ at $\left(u m \cdot x_{0}, \mathbf{t}\right)$ is isomorphic to the direct sum 
of $T_{u}\left(U_{I}\right), T_{m \cdot x_{0}}\left(X_{I, 1}\right)$, and $T_{\mathbf{t}}\left(\mathbb{R}^{d}\right)$, under this identification, it follows from the calculation in Proposition 4.3 of [5] that

$$
\begin{aligned}
& \left(\widetilde{\phi}^{*} \widetilde{g}\right)_{\left(u m \cdot x_{0}, \mathbf{t}\right)} \\
& \quad=\frac{1}{2} \sum_{\chi \in \Upsilon_{I}(\mathbf{G})} \chi(a(\mathbf{t}))^{-2}\left(\left(\operatorname{Int} m^{-1}\right)^{*} h_{\chi}\right)_{u}+\left(\widetilde{g}_{X_{I, 1}}\right)_{m \cdot x_{0}}+\left(d \mathbf{t}^{2}\right)_{\mathbf{t}} .
\end{aligned}
$$

From this, we can conclude that $\phi^{*} g$ is of the form $g_{\mathbf{t}}+d \mathbf{t}^{2}$. Let $\widetilde{g}_{\mathbf{t}}=\Pi^{*} g_{\mathbf{t}}$. Then, from (3.6) we have

$$
\left(\widetilde{g}_{\mathbf{t}}\right)_{u m \cdot x_{0}}=\frac{1}{2} \sum_{\chi \in \Upsilon_{I}(\mathbf{G})} \chi(a(\mathbf{t}))^{-2}\left(\left(\operatorname{Int} m^{-1}\right)^{*} h_{\chi}\right)_{u}+\left(\widetilde{g}_{X_{I, 1}}\right)_{m \cdot x_{0}} .
$$

This shows that the projection

$$
\widetilde{\pi}:\left(\widetilde{Y}_{I, 1, \mathcal{W}}, \widetilde{g}_{\mathbf{t}}\right) \longrightarrow\left(\widetilde{\mathcal{W}},\left.\widetilde{g}_{X_{I, 1}}\right|_{\widetilde{\mathcal{W}}}\right)
$$

is a Riemannian submersion. Let $g_{X, 1}$ be the metric on $V_{I, 1}=\Gamma_{M_{I, 1}} \backslash X_{I, 1}$ corresponding to $\widetilde{g}_{X, 1}$. Since $\Gamma_{I}=\Gamma_{U_{I}} \rtimes \Gamma_{M_{I}}$, the bundle projection

$$
\pi:\left(Y_{I, 1, \mathcal{W}}, g_{\mathbf{t}}\right) \longrightarrow\left(\mathcal{W}, g_{X_{I, 1}} \mid \mathcal{W}\right)
$$

is also a Riemannian submersion and the condition $(\star \star)$ is satisfied.

As is shown in Corollary 4.4 of [5], two left invariant metrics (Int $\left.m^{-1}\right)^{*} d u^{2}$ and $d u^{2}$ have the same volume element. Consequently, it follows from (3.5), (3.7) that

$$
\sqrt{g_{\mathbf{t}}} / \sqrt{g_{\mathbf{0}}}=\prod_{\chi \in \Upsilon_{I}(\mathbf{G})} \chi(a(\mathbf{t}))^{-\operatorname{dim} \mathfrak{u}_{\chi}} / \prod_{\chi \in \Upsilon_{I}(\mathbf{G})} \chi(a(\mathbf{0}))^{-\operatorname{dim} \mathfrak{u}_{\chi}} .
$$

Since

$$
\prod_{\chi \in \Upsilon_{I}(\mathbf{G})} \chi(a(\mathbf{t}))^{-\operatorname{dim} \mathfrak{u}_{\chi}}=\exp \left(-2\left\langle\rho_{I}, H(\mathbf{t})\right\rangle\right)=e^{-2\left|\rho_{I}\right|\left(t+t^{* * *}\right)},
$$

we obtain (3.3), which is the condition $(*)$.

We show that the Riemannian submersion (3.9) is harmonic. For this, it suffices to prove that the Riemannian submersion (3.8) is harmonic. Note that the Lie group $U_{I}$ acts isometrically on $\widetilde{Y}_{I, 1, \mathcal{W}}$ preserving the fibers. Therefore, we can apply H.-S. Wu's theorem on metrically homogeneously fibered submersions to this Riemannian submersion. For precise definitions and detailed information about related concepts we refer to [19, Chapter XV, $\S 6-\S 8]$. 
Let $q=\operatorname{dim} U_{I}$. A $q$-form on $\widetilde{Y}_{I, 1, \mathcal{W}}$ is called fiber null if its restriction to each fiber of (3.8) is 0 . For a horizontal vector field $\nu$ on $\widetilde{Y}_{I, 1, \mathcal{W}}$, we say that a $q$-form $\Psi$ is $\nu$-constant over the fibers if the Lie derivative $\mathcal{L}_{\nu} \Psi$ is fiber null. Consider the pull-back of the left invariant volume form on $U_{I}$ determined by $d u^{2}$ by the projection $U_{I} \times \widetilde{\mathcal{W}} \times\{\mathbf{t}\} \longrightarrow U_{I}$. Then, under the identification $\widetilde{Y}_{I, 1, \mathcal{W}}=U_{I} \times \widetilde{\mathcal{W}} \times\{\mathbf{t}\}$, we obtain a left $U_{I}$-invariant $q$-form $\Psi$ on $\widetilde{Y}_{I, 1, \mathcal{W}}$. This form $\Psi$ is the Haar form on $\widetilde{Y}_{I, 1, \mathcal{W}}$ in the sense of [19, p. 142], and hence $\nu$-constant over the fibers for any horizontal vector field $\nu$, due to $[19$, Theorem 8.3]. Let $\Omega$ be the vertical metric volume form of (3.8) defined as follows. Let $\left\{\xi_{1}, \ldots, \xi_{q}\right\}$ be an orthonormal frame of vertical vector fields on $\widetilde{Y}_{I, 1, \mathcal{W}}$, suitably oriented, and let $\xi_{1}^{*}, \ldots, \xi_{q}^{*}$ be the 1-forms on $\widetilde{Y}_{I, 1, \mathcal{W}}$ such that $\xi_{i}^{*}(\xi)=\widetilde{g}_{\mathbf{t}}\left(\xi_{i}, \xi\right)$ for any vector field $\xi$ on $\widetilde{Y}_{I, 1, \mathcal{W}}$. Put $\Omega=\xi_{1}^{*} \wedge \cdots \wedge \xi_{q}^{*}$. Then $\Omega$ does not depend on the choice of $\left\{\xi_{1}, \ldots, \xi_{q}\right\}$. If $\Omega_{\widetilde{Y}_{I, 1, \mathcal{W}}}, \Omega_{\widetilde{\mathcal{W}}}$ are the volume forms on $\widetilde{Y}_{I, 1, \mathcal{W}}$, $\widetilde{\mathcal{W}}$, respectively, we have $\Omega_{\widetilde{Y}_{I, 1, \mathcal{W}}}=\Omega \wedge \pi^{*} \Omega_{\widetilde{\mathcal{W}}}$. The Riemannian Haar density $([19$, p. 442$]) \widetilde{\omega}$ is by definition the function on $\widetilde{\mathcal{W}}$ given by

$$
\Omega_{u m \cdot x_{0}}=\widetilde{\omega}\left(m \cdot x_{0}\right) \Psi_{u m \cdot x_{0}} .
$$

From (3.7) and (3.10) we have

$$
\widetilde{\omega}\left(m \cdot x_{0}\right)=e^{-2\left(t+t^{* * *}\right)\left|\rho_{I}\right|} / \sqrt{2^{q}} \quad \text { for any } m \in M .
$$

Let $\left(\widetilde{\tau}_{\mathbf{t}}\right)_{u m \cdot x_{0}}$ be the trace of the second fundamental form of the fiber $\widetilde{\pi}^{-1}(m$. $\left.x_{0}\right)$ at $u m \cdot x_{0}$. From H.-S. Wu's theorem $\left(\left[19\right.\right.$, Theorem 6.6]), $\left(\widetilde{\tau}_{\mathbf{t}}\right)_{u m \cdot x_{0}}$ is the horizontal lift of $-(\operatorname{grad}(\log \widetilde{\omega}))_{m \cdot x_{0}}$. Hence $\left(\widetilde{\tau}_{\mathbf{t}}\right)_{x}=0$ for all $x \in \widetilde{Y}_{I, 1, \mathcal{W}}$. This shows that (3.8) is harmonic.

Remark. In the $\mathbb{Q}$-rank $1, \mathbb{R}$-rank $\geq 2$ case, one can show in the same way that each boundary $Y_{j}$ at infinity admits a fiber bundle structure satisfying the conditions $(\star \star),(* *)$.

From (3.3), the mean curvature $\mathcal{K}(t)$ of $Y_{I, j, \mathcal{W}} \times \mathcal{C}_{t}$ is

$$
\mathcal{K}(t)=2\left|\rho_{I}\right| /(n-1)
$$

Let $C_{0}^{\infty}\left(V_{I, j}\right)$ be the space of compactly supported $C^{\infty}$-functions on $V_{I, j}$ and $L^{2}\left(V_{I, j}\right)$ the space of square integrable functions on $V_{I, j}$. We denote by $\bar{\Delta}_{I, j}$ the self-adjoint extension of the Laplacian $\Delta_{I, j}$ on $V_{I, j}$ to $L^{2}\left(V_{I, j}\right)$. We can obtain some information on the spectrum of $\bar{\Delta}$.

Theorem 4. (1) Suppose that

$$
0=c_{I, j, 0}<c_{I, j, 1}<\cdots<c_{I, j, m}<\cdots
$$


are the eigenvalues of $\bar{\Delta}_{I, j}$. Then the following holds.

For any $r \geq 0$, there exists a family $\left\{u_{I, j, m, r, q}\right\}_{q \in \mathbb{N}}$ of compactly supported smooth functions on $\mathcal{E}_{I, j}$ satisfying the following four conditions.

(3.12) For any compact subset of $V$, if we take $q$ sufficiently large, then the support of $u_{I, j, m, r, q}$ lies outside this compact set.

(3.13) For some positive constant $C_{11}$ independent of $q$, we have

$$
\left\|\left(\bar{\Delta}-\left(\left|\rho_{I}\right|^{2}+c_{I, j, m}+r^{2}\right)\right) u_{I, j, m, r, q}\right\| \leq \frac{C_{11}}{q}\left\|u_{I, j, m, r, q}\right\| .
$$

Therefore, for fixed $I, j, m$, every point of $\left[\left|\rho_{I}\right|^{2}+c_{I, j, m}, \infty\right)$ belongs to the spectrum of $\bar{\Delta}$.

(3.14) If $m \neq m^{\prime}$, then

$$
\lim _{\substack{q \rightarrow \infty \\ q^{\prime} \longrightarrow \infty}}\left(u_{I, j, m, r, q}, u_{I, j, m^{\prime}, r^{\prime}, q^{\prime}}\right)=0 .
$$

(3.15) If $\mathbf{P}_{I, j} \cap g \mathbf{P}_{I^{\prime}, j^{\prime}} g^{-1}$ is not a rational parabolic subgroup of $\mathbf{G}$ for any $g \in \mathbf{G}(\mathbb{Q})$, then $\left(u_{I, j, m, r, q}, u_{I^{\prime}, j^{\prime}, m^{\prime}, r^{\prime}, q^{\prime}}\right)=0$.

(2) The bottom of the essential spectrum of $\bar{\Delta}$ is

$$
\min _{I \subset \Upsilon, I \neq \Upsilon}\left|\rho_{I}\right|^{2} .
$$

Remark. For any face $e^{\prime}(\mathbf{P})$ we denote by $\overline{e^{\prime}(\mathbf{P})}$ the closure of $e^{\prime}(\mathbf{P})$ in $\bar{V}$. Then the hypothesis in (3.15) is equivalent to $\overline{e^{\prime}\left(\mathbf{P}_{I, j}\right)} \cap \overline{e^{\prime}\left(\mathbf{P}_{I^{\prime}, j^{\prime}}\right)}=\emptyset$ (see Proposition 9.4 of [7]).

We prove (1) of Theorem 4 in the rest of this section, and (2) in the next section. We denote by \|\|$_{I, j}$ the $L^{2}$-norm on $L^{2}\left(V_{I, j}\right)$.

Proof of Theorem 4 (1). Let $\varphi_{*}$ be an eigenfunction of $\bar{\Delta}_{I, j}$ belonging to the eigenvalue $c_{I, j, m}$. For each $q$, we can find a function $\varphi \in C_{0}^{\infty}\left(V_{I, j}\right)$ such that

$$
\left\|\left(\Delta_{I, j}-c_{I, j, m}\right) \varphi\right\|_{I, j}<\frac{1}{q}\|\varphi\|_{I, j} .
$$

Let $\mathcal{W}$ be a relatively compact open subset containing the support of $\varphi$. For this $\mathcal{W}$, we take $t^{*}=t^{*}(\mathcal{W})$ as in Proposition 3.1, choose $t^{* *} \geq t^{*}$, and identify $A_{I, j}\left(t^{* *}\right) \cdot x_{0}$ with an open cone $\mathcal{C}$ in $\mathbb{R}^{d}$ with $d=r_{\mathbb{Q}}(\mathbf{G})-\# I$. Apply Proposition 1.2 to the embedding $\phi_{I, j, \mathcal{W}}: Y_{I, j, \mathcal{W}} \times \mathcal{C} \longrightarrow V$. From (3.11), we have

$$
\left\|\left(\Delta-\left(\left|\rho_{I}\right|^{2}+c_{I, j, m}+r^{2}\right)\right) f_{\varphi, r, \delta}\right\|<\frac{C_{5}}{q}\left\|f_{\varphi, r, \delta}\right\|
$$


for the function

$$
f_{\varphi, r, \delta}(y, \mathbf{t})=h(\delta \mathbf{t}) e^{-\beta(t)+\sqrt{-1} \cdot r t} \varphi\left(\pi_{I, j}(y)\right),
$$

and any positive number $\delta<\delta(1 / q)$. We take one such $\delta$ and put

$$
u_{I, j, m, r, q}=f_{\varphi, r, \delta} .
$$

Choose the functions $\varphi$ so that $\left\|\varphi_{*}-\varphi\right\|_{I, j}$ are sufficiently small. Then the resultant family $\left\{u_{I, j, m, r, q}\right\}_{q \in \mathbb{N}}$ of compactly supported $C^{\infty}$-functions on $\mathcal{E}_{I, j}$ satisfies the conditions (3.12)-(3.14). In this construction, for fixed $q, q^{\prime}$, we can choose the functions so that the supports of $u_{I, j, m, r, q}$ and $u_{I^{\prime}, j^{\prime}, m^{\prime}, r^{\prime}, q^{\prime}}$ are mutually disjoint for pairs $(I, j),\left(I^{\prime}, j^{\prime}\right)$ such that $\mathbf{P}_{I, j} \cap g \mathbf{P}_{I^{\prime}, j^{\prime}} g^{-1}$ is not a rational parabolic subgroup of $\mathbf{G}$ for any $g \in \mathbf{G}(\mathbb{Q})$. Hence the condition (3.15) is also satisfied. This proves (1) of Theorem 4.

\section{$\S 4$. Constructing Vector Fields}

In this section we prove (2) of Theorem 4 . We construct a vector field on the end of $V$ and use Lemma 1. We recall Borel's construction of fundamental open sets.

Definition 4.1. An open subset $\mathcal{D}$ of $X$ is called a fundamental open set for $\Gamma$ if

$$
X=\Gamma \mathcal{D}
$$

and

(4.2) the set $\{g \in \Gamma \mid g \mathcal{D} \cap \mathcal{D} \neq \emptyset\}$ is finite.

Let $\mathbf{P}=\mathbf{P}_{\emptyset}$ be the standard minimal rational parabolic subgroup and $\mathbf{P}(\mathbb{R})=$ $U A M$ the Langlands decomposition of $\mathbf{P}(\mathbb{R})$ as in (3.1). Since $X=U A M \cdot x_{0}$, any point $x \in X$ can be represented as $x=u a m \cdot x_{0}$ for some $u \in U, a \in A$, $m \in M$. In this representation the $A$-factor $a$ is uniquely determined by $x$. We denote by $\mathcal{A}(x)$ the $A$-factor of $x$. We also recall that $a m=m a$ for any $a \in A$, $m \in M$.

Definition 4.2. For any $t>0$ and relatively compact open subset $\eta$ of $U M$ containing the identity element $e$, the set $\mathfrak{S}_{t \eta}:=\eta A(t) K\left(\right.$ resp. $\mathfrak{S}_{t \eta} \cdot x_{0}=$ $\left.\eta A(t) \cdot x_{0}\right)$ is called a (generalized) Siegel set in $G$ (resp. $X$ ).

We remark that $X$ is also regarded as the quotient $X=\mathbf{G}(\mathbb{R}) / \widetilde{K}$, where $\widetilde{K}$ is the maximal compact subgroup of $\mathbf{G}(\mathbb{R})$ containing $K$. Hence we can consider the action of $\mathbf{G}(\mathbb{R})$ on $X$. Let $z_{1}, \ldots, z_{\lambda}\left(z_{1}=e\right)$ be a complete representative system of $\Gamma \backslash \mathbf{G}(\mathbb{Q}) / \mathbf{P}(\mathbb{Q})$. The following is known. 
Theorem 4.1 ([4]). There exist a positive number $t_{0}<1$ and a relatively compact open subset $\eta$ of $U M$ containing the identity element e such that the set $\Omega=\bigcup_{i=1}^{\lambda} z_{i} \cdot \mathfrak{S}_{t_{0} \eta} \cdot x_{0}$ is a fundamental open set for $\Gamma$.

From now on we fix such $t_{0}$ and $\eta$. The quotient space $V=X / \Gamma$ is obtained by pasting the translated Siegel sets $z_{i} \mathfrak{S}_{t_{0} \eta} \cdot x_{0}, i=1, \ldots, \lambda$, together. In order to describe how these are pasted together, we first decompose $A\left(t_{0}\right)$ as in [16], [17], [18], and [22]. Let $t_{1}$ be any positive number greater than 1 . We put

$$
S_{I}=\left\{a \in A\left(t_{0}\right) \mid \chi(a) \leq t_{1} \quad \text { for all } \chi \in I(\mathbf{G})\right\}
$$

for each nonempty subset $I$ of $\Upsilon$. Then we have

$$
A\left(t_{0}\right)-A\left(t_{1}\right)=\bigcup_{\emptyset \neq I \subset \Upsilon} S_{I}
$$

We remark that the right-hand side is not a disjoint union. Let

$$
\mathcal{S}=\eta A\left(t_{0}\right) \cdot x_{0}=\mathfrak{S}_{t_{0} \eta} \cdot x_{0}, \quad \mathcal{S}_{*}=\eta A\left(t_{1}\right) \cdot x_{0}, \text { and } \mathcal{S}_{I}=\eta S_{I} \cdot x_{0} .
$$

The set $\mathcal{S}_{\Upsilon}$ is relatively compact. We also put

$$
\mathcal{S}_{j}=z_{j} \mathcal{S}, \quad \mathcal{S}_{j *}=z_{j} \mathcal{S}_{*}, \text { and } \mathcal{S}_{j, I}=z_{j} \mathcal{S}_{I}
$$

We have a decomposition

$$
\mathcal{S}_{j}=\mathcal{S}_{j *} \cup\left(\bigcup_{\emptyset \neq I \subset \Upsilon} \mathcal{S}_{j, I}\right) .
$$

Roughly speaking, if $g \mathcal{S}_{i}(g \in \Gamma)$ meets $\mathcal{S}_{j}$ at a point sufficiently far from $x_{0}$, then the intersection $g \mathcal{S}_{i} \cap \mathcal{S}_{j}$ is entirely contained in $\mathcal{S}_{j, I}$ for some nonempty proper subset $I$ of $\Upsilon$. More precisely,

Lemma 4.1 ([4, 12.6], [27, Lemma 2.1], see also [22, Lemmas 2.4, 2.5]). If we take a sufficiently large $t_{1}$, then the following holds:

(1) Suppose that $g \mathcal{S}_{i} \cap \mathcal{S}_{j}$ is nonempty and is relatively compact for some $g \in \Gamma$. Then this intersection is contained in $g \mathcal{S}_{i, \Upsilon} \cap \mathcal{S}_{j, \Upsilon}$.

(2) Suppose that $g \mathcal{S}_{i} \cap \mathcal{S}_{j}$ is not empty nor relatively compact for some $g \in \Gamma$. Let $I$ be the subset of $\Upsilon$ such that $I(\mathbf{G})$ consists of all $\chi \in \Upsilon(\mathbf{G})$ for which $\chi\left(\mathcal{A}\left(\mathcal{S} \cap z_{j}^{-1} g z_{i} \mathcal{S}\right)\right)$ is bounded. Then $z_{j}^{-1} g z_{i} \in\left(U_{I} M_{I}\right)(\mathbb{Q}) \subset P_{I}$ and

$$
g \mathcal{S}_{i} \cap \mathcal{S}_{j} \subset \mathcal{S}_{j, I}, \quad g^{-1} \mathcal{S}_{j} \cap \mathcal{S}_{i} \subset \mathcal{S}_{i, I} .
$$


We also need the following.

Lemma 4.2 ([4, Lemma 12.2]). The union $\bigcup_{a \in A\left(t_{0}\right)} a^{-1} \eta a$ is relatively compact, and hence there exists a positive number $C_{12}$ such that

$$
d_{X}\left(u m a \cdot x_{0}, a \cdot x_{0}\right) \leq C_{12} \quad \text { for } \quad u m \in \eta, u \in U, m \in M, a \in A\left(t_{0}\right),
$$

where $d_{X}$ is the distance on $X$.

Next we consider Busemann functions associated with the geodesic rays corresponding to the edges of the cone $z_{j} A\left(t_{0}\right) \cdot x_{0}$.

Definition 4.3 (cf. [11], [2]). Let $N$ be a complete, simply connected Riemannian manifold of nonpositive sectional curvature and let $d_{N}$ be the distance on $N$.

(1) Two geodesic rays $\gamma_{1}, \gamma_{2}:[0, \infty) \longrightarrow N$ are called asymptotic if the function $t \longmapsto d_{N}\left(\gamma_{1}(t), \gamma_{2}(t)\right)$ is uniformly bounded on $[0, \infty)$. Being asymptotic is an equivalence relation on the set of all geodesic rays in $N$. The equivalence class represented by a geodesic ray $\gamma$ is denoted by $\gamma(\infty)$.

(2) Let $\gamma:[0, \infty) \longrightarrow N$ be a geodesic ray. The Busemann function $h_{\gamma}: N \longrightarrow$ $\mathbb{R}$ associated with $\gamma$ is given by

$$
h_{\gamma}(x)=\lim _{t \rightarrow \infty}\left\{d_{N}(x, \gamma(t))-t\right\} \quad \text { for } x \in N .
$$

For any real number $C$, we call the set $h_{\gamma}^{-1}((-\infty, C))\left(\right.$ resp. $\left.h_{\gamma}^{-1}(C)\right)$ an open horoball (resp. a horosphere) centered at $\gamma(\infty)$, or associated with $\gamma$.

Remark. If a geodesic ray $\gamma_{1}$ is asymptotic to $\gamma_{2}$, then the Busemann function $h_{\gamma_{1}}$ differs to $h_{\gamma_{2}}$ only by an additive constant.

Let $a_{0}$ be the unique point in $A$ such that

$$
\xi\left(a_{0}\right)=t_{0} \quad \text { for all } \xi \in \Upsilon(\mathbf{G}) \text {. }
$$

In other words, $a_{0} \cdot x_{0}$ is the apex of the cone $A\left(t_{0}\right) \cdot x_{0}$. For each $\chi \in \Upsilon(\mathbf{G})$, let $H_{\chi}$ be the unit vector in $\mathfrak{a}^{+}$such that

$$
d \xi\left(H_{\chi}\right)=0 \quad \text { for all } \xi \in \Upsilon(\mathbf{G})-\{\chi\}
$$

and define a geodesic ray $c_{\chi}:[0, \infty) \longrightarrow X$ by

$$
c_{\chi}(t)=a_{0} \exp \left(t H_{\chi}\right) \cdot x_{0} \quad \text { for } t \geq 0 .
$$


These geodesic rays are the edges of $A\left(t_{0}\right) \cdot x_{0}$. Let $c_{j \chi}(t)=z_{j} c_{\chi}(t)$. For each $j$ the edges of the cone $z_{j} A\left(t_{0}\right) \cdot x_{0}$ are $c_{j \chi}, \chi \in \Upsilon(\mathbf{G})$. Let $h_{j \chi}$ be the Busemann function associated with $c_{j \chi}$. We remark that if $\xi \neq \chi$, then the geodesic ray $c_{j \xi}$ is not asymptotic to $g c_{i \chi}$ for any $g \in \Gamma, i \in\{1, \ldots, \lambda\}$. This can be seen, for example, as follows. Let $I, J$ be the subsets of $\Upsilon$ such that $I(\mathbf{G})=\Upsilon(\mathbf{G})-\{\chi\}$, $J(\mathbf{G})=\Upsilon(\mathbf{G})-\{\xi\}$. If $\chi \neq \xi$, then we have $I \neq J$. From Mostow's lemma (cf. $\left[18\right.$, Lemma 5.7]), the isotropy subgroup of $c_{\chi}(\infty)\left(\right.$ resp. $\left.c_{\xi}(\infty)\right)$ is $\mathbf{P}_{I}(\mathbb{R})$ (resp. $\left.\mathbf{P}_{J}(\mathbb{R})\right)$. Suppose that $c_{j \xi}$ is asymptotic to $g c_{i \chi}$. Then, $\mathbf{P}_{I}$ is conjugate to $\mathbf{P}_{J}$ by $\left[30,3.1 .9\right.$ Theorem]. On the other hand, $\mathbf{P}_{I}$ and $\mathbf{P}_{J}$ are standard rational parabolic subgroups of $\mathbf{G}$ with $I \neq J$, and hence they are not conjugate to each other ([3, V. 21.12 Proposition] $)$, which is a contradiction. When $c_{j \chi}$ is asymptotic to $g c_{i \chi}$ for some $g \in \Gamma$, we have

$$
h_{j \chi}(g x)=h_{i \chi}(x)+s_{i j, \chi} \quad \text { for all } x \in X,
$$

where $s_{i j, \chi}$ is a constant depending on $i, j, \chi$ but not on $g$ (cf. [22, Proposition $3.3]$ ).

For each $\chi \in \Upsilon(\mathbf{G})$, we renormalize the Busemann functions $h_{1 \chi}, \ldots, h_{\lambda \chi}$ as follows. For each $i \in\{1, \ldots, \lambda\}$, let $q=q(i) \in\{1, \ldots, \lambda\}$ be the smallest index such that there exists an element $g$ of $\Gamma$, for which the geodesic ray $g c_{q \chi}$ is asymptotic to $c_{i \chi}$. Let

$$
\widetilde{h}_{i \chi}=h_{i \chi}-s_{q(i) i, \chi} .
$$

Then we have

Lemma 4.3 ([22, Lemma 3.4]). Suppose that $g c_{i \chi}$ is asymptotic to $c_{j \chi}$ for $g \in \Gamma$. Then

$$
\widetilde{h}_{j \chi}(g x)=\widetilde{h}_{i \chi}(x) \quad \text { for all } x \in X .
$$

By adding a constant to all the functions $\widetilde{h}_{i \chi}$ simultaneously if necessary, we can also assume the following for each $j$.

$$
\left(\bigcap_{\chi \in \Upsilon(\mathbf{G})} \widetilde{h}_{j \chi}^{-1}(0)\right) \cap z_{j} A \cdot x_{0}=\left\{z_{j} b_{j} \cdot x_{0}\right\}, \quad b_{j} \in A\left(t_{1}\right) .
$$

By using these results, we consider another decomposition of each $\mathcal{S}_{j}$. In this paragraph we fix one $j$. Let

$$
r_{j \chi}=\chi\left(b_{j}\right) \quad\left(>t_{1}\right)
$$

for each $\chi \in \Upsilon(\mathbf{G})$. Let

$$
F_{j *}=\left\{a \cdot x_{0} \mid \xi(a)>r_{j \xi} \quad \text { for all } \xi \in \Upsilon(\mathbf{G})\right\}
$$




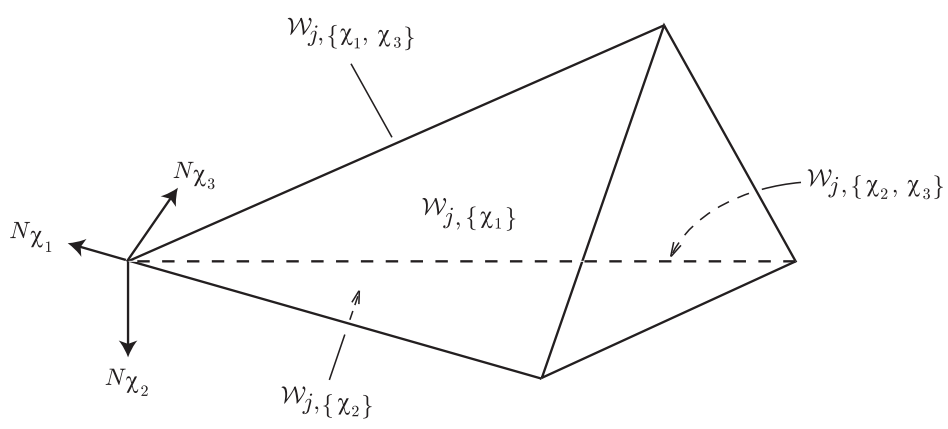

Figure 5. $F_{j *}$ in the $\mathbb{Q}$-rank 3 case: $\Upsilon(\mathbf{G})=\left\{\chi_{1}, \chi_{2}, \chi_{3}\right\}$.

be the cone in $A\left(t_{1}\right) \cdot x_{0}$ with apex $b_{j} \cdot x_{0}$. For each nonempty subset $I$ of $\Upsilon$, we define a subset $\mathcal{W}_{j, I}$ of $A \cdot x_{0}$ by

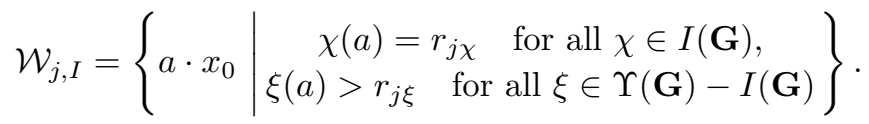

These $\mathcal{W}_{j, I}$ form the boundary of the cone $F_{j *}$ (see Figure 5 ). We regard $A \cdot x_{0}$ as the $r_{\mathbb{Q}}(\mathbf{G})$-dimensional Euclidean space. For each $\chi \in \Upsilon(\mathbf{G})$, let $N_{\chi}$ be the outer unit normal vector (in $\mathbb{R}^{r_{\mathbb{Q}}(\mathbf{G})}$ ) of the maximal face $\mathcal{W}_{j,\{\chi\}}$ of $F_{j *}$. We put

$$
F_{j, I}=\left\{b \cdot x_{0}+\sum_{\xi \in I(\mathbf{G})} t_{\xi} N_{\xi} \in A\left(t_{0}\right) \cdot x_{0} \mid \begin{array}{c}
b \cdot x_{0} \in \mathcal{W}_{j, I}, \\
t_{\xi} \geq 0 \quad \text { for all } \xi \in I(\mathbf{G})
\end{array}\right\}
$$

for each nonempty subset $I$ of $\Upsilon$. Then we have

$$
A\left(t_{0}\right) \cdot x_{0}=F_{j *} \cup\left(\bigcup_{\emptyset \neq I \subset \Upsilon} F_{j, I}\right) .
$$

Let

$$
\widetilde{\mathcal{S}}_{j *}=z_{j} \eta F_{j *}, \quad \widetilde{\mathcal{S}}_{j, I}=z_{j} \eta F_{j, I} .
$$

Then we have a decomposition

$$
\mathcal{S}_{j}=\widetilde{\mathcal{S}}_{j *} \cup\left(\bigcup_{\emptyset \neq I \subset \Upsilon} \widetilde{\mathcal{S}}_{j, I}\right) .
$$




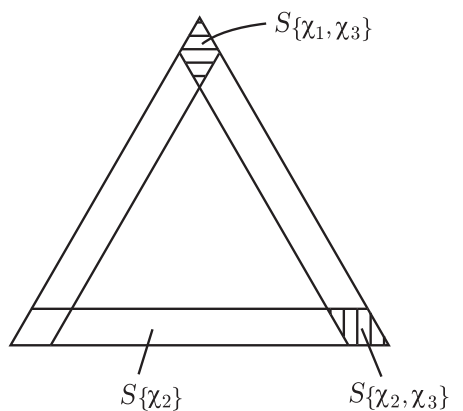

The decomposition by (4.3).

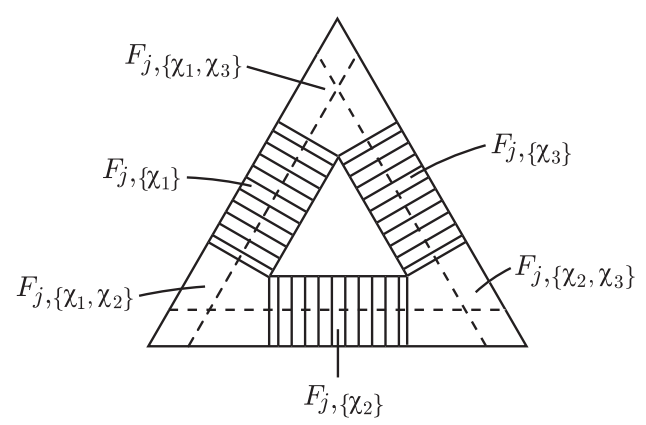

The decomposition by (4.6).

Figure 6 . The section of $A\left(t_{0}\right) \cdot x_{0}$ by a hyperplane (in $A \cdot x_{0}$ ) transverse to the geodesic ray $\exp (t \rho /|\rho|) \cdot x_{0}: \mathbb{Q}$-rank $=3, \Upsilon(\mathbf{G})=\left\{\chi_{1}, \chi_{2}, \chi_{3}\right\}$.

We remark (see Figure 6) that $\widetilde{\mathcal{S}}_{j *} \subset \mathcal{S}_{j *}$ and

$$
\widetilde{\mathcal{S}}_{j *} \cup\left(\bigcup_{I \neq \Upsilon, \emptyset} \widetilde{\mathcal{S}}_{j, I}\right) \subset \mathcal{S}_{j}-\mathcal{S}_{j, \Upsilon} .
$$

By (4.3), (4.6) we obtained $(\lambda+1)$ different decompositions of $A \cdot x_{0}$. In this paragraph we fix a nonempty subset $I$, and compare the locations of various $\mathcal{W}_{j, I}$ when $j$ runs through 1 to $\lambda$. We consider them in the Euclidean space $A \cdot x_{0}$. First of all, let $\mathcal{H}_{j, I}$ be the $\left(r_{\mathbb{Q}}(\mathbf{G})-\# I\right)$-dimensional plane containing $\mathcal{W}_{j, I}$. Then these planes $\mathcal{H}_{j, I}$ are mutually parallel. Let $v_{i j}=$ $b_{j} \cdot x_{0}-b_{i} \cdot x_{0}$. From the definition of $\mathcal{W}_{j, I}$, the set $\left\{\widetilde{h}_{j \xi}\right\}_{\xi \in \Upsilon(\mathbf{G})-I(\mathbf{G})}$ of renormalized Busemann functions can be used as a coordinate system on $\mathcal{H}_{j, I}$. More precisely, if $a \cdot x_{0}, a^{\prime} \cdot x_{0} \in \mathcal{H}_{j, I}$ and

$$
\widetilde{h}_{j \xi}\left(z_{j} a \cdot x_{0}\right)=\widetilde{h}_{j \xi}\left(z_{j} a^{\prime} \cdot x_{0}\right) \quad \text { for all } \xi \in \Upsilon(\mathbf{G})-I(\mathbf{G}),
$$

then $a=a^{\prime}$. Moreover, from the choice of $b_{1}, \ldots, b_{\lambda}$ in (4.5), if $a \cdot x_{0} \in \mathcal{H}_{j, I}$, $a^{\prime \prime} \cdot x_{0} \in \mathcal{H}_{i, I}$ and

$$
\widetilde{h}_{j \xi}\left(z_{j} a \cdot x_{0}\right)=\widetilde{h}_{i \xi}\left(z_{i} a^{\prime \prime} \cdot x_{0}\right) \quad \text { for all } \xi \in \Upsilon(\mathbf{G})-I(\mathbf{G}),
$$

we have $a \cdot x_{0}=a^{\prime \prime} \cdot x_{0}+v_{i j}$. Let

$$
C_{13}=C_{12}+\max _{1 \leq j \leq \lambda} d_{X}\left(x_{0}, b_{j} \cdot x_{0}\right)
$$


Lemma 4.4. Suppose that

$$
x=z_{i} u m a \cdot x_{0} \in \mathcal{S}_{i}, \quad x^{\prime}=z_{j} u^{\prime} m^{\prime} a^{\prime} \cdot x_{0} \in \mathcal{S}_{j}, \quad d_{X}\left(x_{0}, x^{\prime}\right)>C_{13},
$$

and $g x=x^{\prime}$ for some $g \in \Gamma$. Then the following holds:

(1) For some proper subset $I$ of $\Upsilon, x \in \mathcal{S}_{i, I}$ and $x^{\prime} \in \mathcal{S}_{j, I}$.

(2) The line in $A \cdot x_{0}$ through the two points $a \cdot x_{0}+v_{i j}$ and $a^{\prime} \cdot x_{0}$ is perpendicular to $\mathcal{H}_{j, I}$.

Proof. Since $x^{\prime} \notin \mathcal{S}_{j, \Upsilon}$, (1) follows from Lemma 4.1. Moreover we have $z_{j}^{-1} g z_{i} \in\left(U_{I} M_{I}\right)(\mathbb{Q}) \subset P_{I}$. Then the geodesic ray $g c_{i \xi}$ is asymptotic to $c_{j \xi}$ for all $\xi \in \Upsilon(\mathbf{G})-I(\mathbf{G})\left(\left[18\right.\right.$, Proposition 5.9]). Let $\widetilde{c}_{i \xi}$ (resp. $\widetilde{c}_{j \xi}$ ) be the geodesic ray in $A\left(t_{0}\right) \cdot x_{0}$ which corresponds to $\mathcal{W}_{i,\{\xi\}}\left(\operatorname{resp} . \mathcal{W}_{j,\{\xi\}}\right)$. Then $\widetilde{h}_{i \xi}$ (resp. $\widetilde{h}_{j, \xi}$ ) is the Busemann function with respect to $z_{i} \widetilde{c}_{i \xi}\left(\right.$ resp. $\left.z_{j} \widetilde{c}_{j \xi}\right)$. Note that the values of the Busemann functions with respect to $\widetilde{c}_{i \xi}, \widetilde{c}_{j \xi}$ are invariant under the action of $U M$. Hence we have

$$
\widetilde{h}_{i \xi}\left(z_{i} u m a \cdot x_{0}\right)=\widetilde{h}_{i \xi}\left(z_{i} a \cdot x_{0}\right), \quad \widetilde{h}_{j \xi}\left(z_{j} u^{\prime} m^{\prime} a^{\prime} \cdot x_{0}\right)=\widetilde{h}_{j \xi}\left(z_{j} a^{\prime} \cdot x_{0}\right) .
$$

On the other hand, from Lemma 4.3, we have

$$
\widetilde{h}_{i \xi}\left(z_{i} u m a \cdot x_{0}\right)=\widetilde{h}_{j \xi}\left(g z_{i} u m a \cdot x_{0}\right)=\widetilde{h}_{j \xi}\left(z_{j} u^{\prime} m^{\prime} a^{\prime} \cdot x_{0}\right)
$$

for $\xi \in \Upsilon(\mathbf{G})-I(\mathbf{G})$. It follows from (4.8) that

$$
\widetilde{h}_{i \xi}\left(z_{i} a \cdot x_{0}\right)=\widetilde{h}_{j \xi}\left(z_{j} a^{\prime} \cdot x_{0}\right)
$$

for $\xi \in \Upsilon(\mathbf{G})-I(\mathbf{G})$. We take the unique point $b \cdot x_{0}$ (resp. $\left.b^{\prime} \cdot x_{0}\right)$ in the $\left(r_{\mathbb{Q}}(\mathbf{G})-\# I\right)$-dimensional plane $\mathcal{H}_{i, I}$ (resp. $\mathcal{H}_{j, I}$ ) such that the line through $a \cdot x_{0}\left(\operatorname{resp} . a^{\prime} \cdot x_{0}\right)$ and $b \cdot x_{0}\left(\operatorname{resp} . b^{\prime} \cdot x_{0}\right)$ is perpendicular to this plane. Then we have

$$
\widetilde{h}_{i \xi}\left(z_{i} b \cdot x_{0}\right)=\widetilde{h}_{i \xi}\left(z_{i} a \cdot x_{0}\right), \quad \widetilde{h}_{j \xi}\left(z_{j} b^{\prime} \cdot x_{0}\right)=\widetilde{h}_{j \xi}\left(z_{j} a^{\prime} \cdot x_{0}\right)
$$

for all $\xi \in \Upsilon(\mathbf{G})-I(\mathbf{G})$. From (4.9), (4.10), we obtain

$$
\widetilde{h}_{i \xi}\left(z_{i} b \cdot x_{0}\right)=\widetilde{h}_{j \xi}\left(z_{j} b^{\prime} \cdot x_{0}\right) \quad \text { for all } \xi \in \Upsilon(\mathbf{G})-I(\mathbf{G})
$$

Therefore, $b^{\prime} \cdot x_{0}=b \cdot x_{0}+v_{i j}$ and $a^{\prime}-\left(a+v_{i j}\right)$ is perpendicular to $\mathcal{H}_{j, I}$.

We can now construct a vector field on $V$. 
For each $j$, we regard $A \cdot x_{0}$ as the Euclidean space $\mathbb{R}^{r_{\mathbb{Q}}(\mathbf{G})}$ with origin $O=b_{j} \cdot x_{0}$. Let $\left(r, \theta_{1}, \ldots, \theta_{r_{Q}(\mathbf{G})-1}\right)$ be the polar coordinate of $\mathbb{R}^{r_{Q}(\mathbf{G})}$. We define a vector field $Z_{j}^{\prime \prime}$ on the (open) cone $F_{j *}$ with apex $O$ by

$$
\left(Z_{j}^{\prime \prime}\right)_{a \cdot x_{0}}=-\left(\frac{\partial}{\partial r}\right)_{a \cdot x_{0}} \quad \text { for all } a \cdot x_{0} \in F_{j *} .
$$

Let $A_{j}^{\prime}\left(t_{0}\right) \cdot x_{0}$ be the region obtained from $A\left(t_{0}\right) \cdot x_{0}$ by deleting a closed ball of radius $R_{j}$ with center $O$, where $R_{j}$ is an arbitrary number larger than the distance from $O$ to the apex $a_{0} \cdot x_{0}$ of the cone $A\left(t_{0}\right) \cdot x_{0}$. We extend the vector field $Z_{j}^{\prime \prime}$ to $A_{j}^{\prime}\left(t_{0}\right) \cdot x_{0} \cap F_{j, I}$ for each nonempty subset $I$ of $\Upsilon$ : We define $\left(Z_{j}^{\prime \prime}\right)_{x}$ to be the vector obtained by the parallel translation in $\mathbb{R}^{r_{\mathbb{Q}}(\mathbf{G})}$ from $\left(Z_{j}^{\prime \prime}\right)_{b \cdot x_{0}}$ when

$$
x=b \cdot x_{0}+\sum_{\xi \in I(\mathbf{G})} t_{\xi} N_{\xi} \in A_{j}^{\prime}\left(t_{0}\right) \cdot x_{0} \cap F_{j, I}, \quad b \cdot x_{0} \in \mathcal{W}_{j, I} .
$$

Further we define $\left(Z_{j}^{\prime \prime}\right)_{u m a \cdot x_{0}}$ for $u m a \cdot x_{0} \in \eta A_{j}^{\prime}\left(t_{0}\right) \cdot x_{0}$ to be the horizontal vector which is mapped to the vector $\left(Z_{j}^{\prime \prime}\right)_{a \cdot x_{0}}$ by the Riemannian submersion $\eta A_{j}^{\prime}\left(t_{0}\right) \cdot x_{0} \longrightarrow A_{j}^{\prime}\left(t_{0}\right) \cdot x_{0}$. We have thus obtained a vector field $Z_{j}^{\prime \prime}$ on $\eta A_{j}^{\prime}\left(t_{0}\right) \cdot x_{0}$ which is smooth on the complement of $\bigcup_{I \neq \emptyset, \Upsilon} \eta \mathcal{W}_{j, I}$.

By using the differential of the left translation $L_{z_{j}}$, we define a vector field $Z_{j}^{\prime}$ on $\mathcal{S}_{j}^{\prime}:=z_{j} \eta A_{j}^{\prime}\left(t_{0}\right) \cdot x_{0}$ by

$$
\left(Z_{j}^{\prime}\right)_{z_{j} u m a \cdot x_{0}}=d L_{z_{j}}\left(\left(Z_{j}^{\prime \prime}\right)_{u m a \cdot x_{0}}\right) \quad \text { for } u m a \cdot x_{0} \in \eta A_{j}^{\prime}\left(t_{0}\right) \cdot x_{0} .
$$

From Lemmas 4.1, 4.4, these vector fields $Z_{1}^{\prime}, \ldots, Z_{\lambda}^{\prime}$ are well patched together to give a vector field $Z^{\prime}$ on the complement of some compact subset of $V$. By using a suitable cut-off function, we can extend this $Z^{\prime}$ to a vector field $Z$ on $V$.

We recall that $\Pi: X \longrightarrow V$ is the natural projection. Let $v_{0}=\Pi\left(x_{0}\right)$ and let $B_{R}\left(v_{0}\right)$ be the closed geodesic ball in $V$ of radius $R>0$ around $v_{0}$. If we take a sufficiently large $R$, then $Z$ coincides with $Z^{\prime}$ on $V-B_{R}\left(v_{0}\right)$ due to Lemma 4.2. Take a submanifold with smooth boundary including $B_{R}\left(v_{0}\right)$, and let $V_{R}$ be its complement. (We can find such a submanifold, for example, by using the exhaustion function constructed in [27]). We have $|Z| \equiv 1$ on the open submanifold $V_{R}$ of $V$. Let $W$ be the image under $\Pi$ of the union of $z_{j} \eta \partial F_{j *}$ and $z_{j} \eta\left(\partial F_{j, I}-\partial\left(A\left(t_{0}\right) \cdot x_{0}\right)\right), 1 \leq j \leq \lambda ; I \neq \emptyset, \Upsilon$, where $\partial$ means the boundary. Since $\bigcup_{j=1}^{\lambda} z_{j} \eta A\left(t_{0}\right) \cdot x_{0}$ is a fundamental open set for $\Gamma, W \cap V_{R}$ is the union of a finite number of closed submanifolds of $V_{R}$ of codimension 1. From the construction of $Z$, for any $v \in W, Z(v)$ is tangent to $W$. The 
complement $V_{R}-W$ is a disjoint union of a finite number of open submanifolds, say $V_{1}, \ldots, V_{s}$. For any given $u \in C_{0}^{\infty}\left(V_{R}\right)$, we have the following: If $v \in \partial V_{i}$, then $u(v)=0$ or $Z(v)$ is tangent to $\partial V_{i}$. Hence, if we find a positive constant $C$ such that $\operatorname{div} Z \geq C$ on each $V_{i}$, we can apply the similar argument in the proof of Lemma 1 to each $V_{i}$ to yield

$$
\frac{C^{2}}{4}\left(\int_{V_{i}}|u|^{2}\right) \leq \int_{V_{i}}|\operatorname{grad} u|^{2} .
$$

By taking the sum, we obtain

$$
\frac{C^{2}}{4}\left(\int_{V_{R}}|u|^{2}\right) \leq \int_{V_{R}}|\operatorname{grad} u|^{2}
$$

and the essential spectrum of $V_{R}$ is contained in $\left[C^{2} / 4, \infty\right)$. Therefore, it suffices to find such a constant.

Lemma 4.5. For any $\varepsilon>0$, there exists a number $R(\varepsilon)>0$ such that the following holds. If $R \geq R(\varepsilon)$, then we have

$$
\operatorname{div} Z \geq 2\left(\min _{I \neq \Upsilon}\left|\rho_{I}\right|-\varepsilon\right) \quad \text { on } V-B_{R}\left(v_{0}\right) .
$$

Proof. It suffices to show the following for $R \geq R(\varepsilon)$ :

$$
\operatorname{div} Z \geq 2\left(\min _{I \subset J \neq \Upsilon}\left|\rho_{J}\right|-\varepsilon\right) \quad \text { on each } \Pi\left(\widetilde{\mathcal{S}}_{j, I}\right)-B_{R}\left(v_{0}\right) .
$$

For this, it suffices to estimate $\operatorname{div} Z_{j}^{\prime \prime}$ on $\eta\left(F_{j, I} \cap A_{j}^{\prime}\left(t_{0}\right) \cdot x_{0}\right)$.

Let $d=r_{\mathbb{Q}}(\mathbf{G})-\# I$. We regard $A \cdot x_{0}$ as the Euclidean space $\mathbb{R}^{r_{\mathbb{Q}}(\mathbf{G})}$ with the origin $b_{j} \cdot x_{0}$. Let us consider the $d$-dimensional subspace containing $\mathcal{W}_{j, I}$. Let $\left(r, \theta_{1}, \ldots, \theta_{d-1}\right)$ be its polar coordinate system, where

$$
0 \leq r ; 0 \leq \theta_{1}, \ldots, \theta_{d-2} \leq \pi / 2 ; 0 \leq \theta_{d-1} \leq 2 \pi
$$

and the last angle $\theta_{d-1}$ is counted from the ray

$$
\left\{\exp \left(t \rho_{I} /\left|\rho_{I}\right|\right) b_{j} \cdot x_{0} \mid t \geq 0\right\}
$$

in $\mathcal{W}_{j . I}$. (The ray is represented as $(t, \pi / 2, \ldots, \pi / 2,0)$ in this coordinate system.) We take a coordinate system $\left(\nu_{1}, \ldots, \nu_{n-d}\right)$ of the space $U_{I} M_{I} \cdot x_{0}$, which is diffeomorphic to the $(n-d)$-dimensional Euclidean space. Then $\left(r, \theta_{1}, \ldots, \theta_{d-1}, \nu_{1}, \ldots, \nu_{n-d}\right)$ is a coordinate system of $X$. We can assume that this coordinate system is compatible with the orientation of $X$. Let

$$
h\left(\nu_{1}, \ldots, \nu_{n-d}\right) d \nu_{1} \wedge \cdots \wedge d \nu_{n-d}
$$


be the volume element of $U_{I} M_{I} \cdot x_{0}$. Then, from [5, Corollary 4.4], the volume element of $X$ is given by

$$
\sqrt{g} d r \wedge d \theta_{1} \wedge \cdots \wedge d \theta_{d-1} \wedge d \nu_{1} \wedge \cdots \wedge d \nu_{n-d},
$$

where

$$
\begin{gathered}
\sqrt{g}=r^{d-1} e^{-2 r\left|\rho_{I}\right| \sin \theta_{1} \cdots \sin \theta_{d-1}} f\left(\theta_{1}, \ldots, \theta_{d-1}\right) h\left(\nu_{1}, \ldots, \nu_{n-d}\right), \\
f\left(\theta_{1}, \ldots, \theta_{d-1}\right)=e^{-2\left\langle\rho_{I}, \log b_{j}\right\rangle} \prod_{k=1}^{d-2} \sin ^{d-k-1} \theta_{k} .
\end{gathered}
$$

In this coordinate system, $Z_{j}^{\prime \prime}$ is represented as

$$
Z_{j}^{\prime \prime}=-1 \cdot \frac{\partial}{\partial r}
$$

Consequently, we have

$$
\operatorname{div} Z_{j}^{\prime \prime}=\frac{1}{\sqrt{g}} \frac{\partial}{\partial r}(-\sqrt{g})=-\frac{d-1}{r}+2\left|\rho_{I}\right| \sin \theta_{1} \cdots \sin \theta_{d-1} .
$$

Thus we have, for $x=u m a b_{j} \cdot x_{0} \in \eta\left(F_{j, I} \cap A_{j}^{\prime}\left(t_{0}\right) \cdot x_{0}\right), u \in U_{I}, m \in M_{I}, a \in A_{I}$,

$$
\left(\operatorname{div} Z_{j}^{\prime \prime}\right)(x)=-\frac{d-1}{|\log a|}+2\left\langle\rho_{I}, \frac{\log a}{|\log a|}\right\rangle \geq-\frac{d-1}{|\log a|}+2 \min _{I \subset J \neq \Upsilon}\left|\rho_{J}\right| .
$$

There exists a positive constant $C_{14}(I)$ determined by the angle between $\rho$ and $\mathcal{W}_{j, I}$ such that the following holds: If

$$
R \geq R_{j}(\varepsilon):=\left|\log b_{j}\right|+C_{12}+C_{14}(I) \cdot \frac{d-1}{2 \varepsilon},
$$

then

$$
\begin{aligned}
& |\log a| \geq(d-1) /(2 \varepsilon) \quad \text { for } x=u m a b_{j} \cdot x_{0} \in \eta\left(F_{j, I} \cap A_{j}^{\prime}\left(t_{0}\right) \cdot x_{0}\right), \\
& u \in U_{I}, m \in M_{I}, a \in A_{I} \text { with } \Pi(x) \in V_{R} .
\end{aligned}
$$

If we put

$$
R(\varepsilon)=\max _{1 \leq j \leq \lambda, I \neq \Upsilon} R_{j}(\varepsilon),
$$

then (4.12) follows from (4.19), Lemma 4.2, and the triangle inequality.

Proof of Theorem 4 (2). For any $\varepsilon>0$, take a submanifold $V_{R}$ with $R \geq$ $R(\varepsilon)$. Then the essential spectrum of $V_{R}$ is contained in $\left[\left(\min _{I \neq \Upsilon}\left|\rho_{I}\right|-\varepsilon\right)^{2}, \infty\right)$ 
due to Lemma 4.5. Hence, from the decomposition principle, the essential spectrum of $V$ is contained in

$$
\bigcap_{0<\varepsilon \ll 1}\left[\left(\min _{I \neq \Upsilon}\left|\rho_{I}\right|-\varepsilon\right)^{2}, \infty\right)=\left[\min _{I \neq \Upsilon}\left|\rho_{I}\right|^{2}, \infty\right) .
$$

On the other hand, we have already seen (in Section 3) that $\left[\min _{I \neq \Upsilon}\left|\rho_{I}\right|^{2}, \infty\right.$ ) belongs to the essential spectrum of $V$. Therefore, $\min _{I \neq \Upsilon}\left|\rho_{I}\right|^{2}$ is the bottom of the essential spectrum of $V$.

Remark. After this paper was written, the author was informed that our construction in this section might be related to the construction in [28].

\section{§5. Manifolds with Corners at Infinity}

In this section we discuss some other consequences of Sections 1 and 2.

For any given compact manifold $M$ with boundary $\partial M$, we can attach $\partial M \times[0, \infty)$ to the boundary to produce a complete Riemannian manifold $V$ and control the bottom of the essential spectrum of $V$. Let $Y_{1}, \ldots, Y_{s}$ be the connected components of $\partial M$. We choose a metric on each $Y_{j} \times[0, \infty)$ so that the condition $(*)$ in the introduction is satisfied. For example, if

$$
\alpha_{j}(t)=e^{-t^{1+a}}, \quad a>0,
$$

then, from Lemma 1 or Theorem 2, the essential spectrum of $V$ is empty. If

$$
\alpha_{j}(t)=e^{ \pm 2 \sqrt{c} t},
$$

then, from Theorem 1 (1) and Lemma 1 (or Proposition 1), the essential spectrum of $V$ is the half-line $[c, \infty)(c \geq 0)$. In particular, if $\alpha_{j}(t)=e^{-2 \sqrt{c} t}$ for some $j, V$ has a shrinking end. When $Y_{j}$ admits a fiber bundle structure $Y_{j} \longrightarrow B_{j}$ satisfying the conditions $(\star \star),(* *)$ in the introduction, we can give an additional structure to the essential spectrum of $V$ : We first deform the metric on $Y_{j}$ to the metric of such a Riemannian submersion in the part $Y_{j} \times[0,1]$, and then apply Theorem $1(2)$ to $Y_{j} \times(1, \infty)$. The essential spectrum of $V$ contains a union of half-lines parametrized by the eigenvalues of $B_{j}$.

Theorem 1 can be applied to complete manifolds canonically obtained from manifolds with corners. Following [24], a manifold $W$ with corners is a topological manifold with boundary equipped with an embedding $\iota: W \hookrightarrow \widetilde{W}$ into a closed $C^{\infty}$-manifold for which there exists a finite collection of smooth functions $\rho_{i}$ on $\widetilde{W}, i \in I$, such that

$$
\iota(W)=\left\{x \in \widetilde{W} \mid \rho_{i}(x) \geq 0, i \in I\right\}
$$




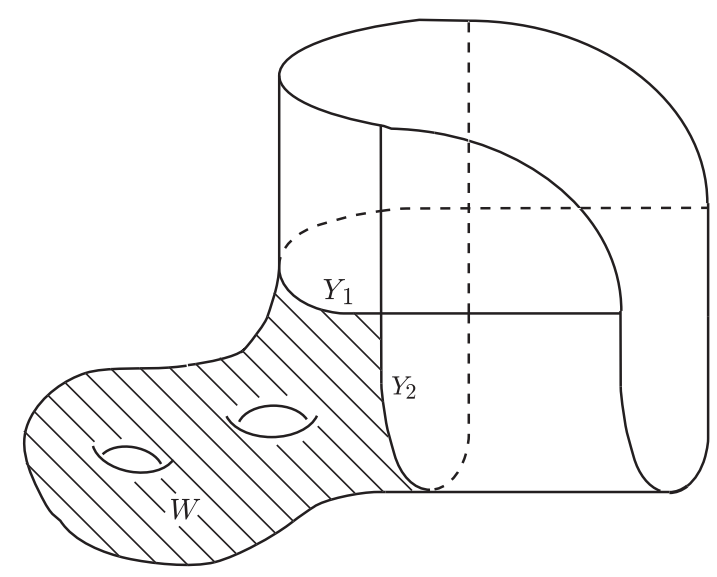

Figure 7. A manifold with corners at infinity: the case $\# I=2$.

and for each subset $J \subset I$, the differentials $d \rho_{i}, i \in J$, are linearly independent at each point $x \in \widetilde{W}$ where all $\rho_{i}, i \in J$ vanish. We identify $W$ with the image $\iota(W)$. Let

$$
Y_{i}=W \cap\left\{x \in \widetilde{W} \mid \rho_{i}(x)=0\right\}
$$

for each $i \in I$. Then the boundary $\partial W$ of $W$ is the union of the hypersurfaces $Y_{i}, i \in I$. For any subset $J=\left\{i_{1}, \ldots, i_{k}\right\} \subset I$, we put

$$
Y_{J}=Y_{i_{1} \cdots i_{k}}=Y_{i_{1}} \cap \cdots \cap Y_{i_{k}} .
$$

We say that $Y_{J}$ is a corner of codimension $k$. We assume that $W$ is endowed with a metric which is a product on a neighborhood of the form $(-\varepsilon, 0]^{k} \times Y_{J}$ for each corner $Y_{J}$ of codimension $k$.

In this situation, we can enlarge $W$ as follows: We first glue half-cylinders $Y_{i} \times[0, \infty)$ to the codimension 1 corners $Y_{i}$ to obtain the space $W_{1}$. Next we glue $Y_{i j} \times[0, \infty)^{2}$ to each codimension 2 corner $Y_{i j}$ to get the space $W_{2}$. After repeating this procedure, we finally fill $W_{\# I-1}$ with $Y_{I} \times[0, \infty)^{\# I}$ at the codimension \#I corner $Y_{I}$ and obtain a complete manifold $V$. Let us call this $V$ the complete manifold obtained from $W$ by gluing cylinders successively to boundary components, or briefly the complete manifold canonically obtained from $W$.

Proof of Corollary 1. Apply Theorem 1 (1) to the part $Y_{I} \times[0, \infty)^{\# I}$ under the condition that the mean curvature $\mathcal{K}(t)$ is identically zero. 
We consider the case $\# I=2$ further. Let $W$ be an $n$-dimensional manifold with boundary $\partial W=Y_{1} \cup Y_{2}$, where $Y_{1}$ and $Y_{2}$ are $(n-1)$-dimensional compact manifolds such that $Y_{12}=Y_{1} \cap Y_{2}$ is the boundary of both $Y_{1}$ and $Y_{2}$. By deforming its metric (in a compact region) if necessary, we may assume that $Y_{12}$ is orthogonal to both $Y_{1}$ and $Y_{2}$, and that the metric of $W$ is a product near all $Y_{1}, Y_{2}$ and $Y_{12}$. We first glue $Y_{1} \times[0, \infty), Y_{2} \times[0, \infty)$ to $Y_{1}, Y_{2}$, respectively, and then we attach $Y_{12} \times[0, \infty)^{2}$ to $Y_{12}$ as in Figure 7 to get a complete manifold $V$. Let

$$
\hat{Y}_{1}=Y_{1} \cup_{Y_{12}}\left(Y_{12} \times[0, \infty)\right)
$$

be the manifold obtained from $Y_{1}$ by attaching $Y_{12} \times[0, \infty)$ to $Y_{12}$, and let

$$
\hat{Y}_{2}=Y_{2} \cup_{Y_{12}}\left(Y_{12} \times[0, \infty)\right) .
$$

Then $\hat{Y}_{1}, \hat{Y}_{2}$ have infinite volume and $Y_{12}=\hat{Y}_{1} \cap \hat{Y}_{2}$,

$$
V=W \cup\left(\hat{Y}_{1} \times[0, \infty)\right) \cup\left(\hat{Y}_{2} \times[0, \infty)\right)
$$

Suppose that there exist eigenvalues of $\hat{Y}_{1}$ and $\hat{Y}_{2}$. Then we can apply Theorem 1 (2) to the three parts $\hat{Y}_{1} \times[0, \infty), \hat{Y}_{2} \times[0, \infty)$, and $Y_{12} \times[0, \infty)^{2}$ under the condition that the mean curvature $\mathcal{K}(t)$ is identically zero. Let $c_{j, 0}<c_{j, 1}<\cdots<c_{j, m}<\cdots$ be the eigenvalues of $\hat{Y}_{1}$ if $j=1, \hat{Y}_{2}$ if $j=2, Y_{12}$ if $j=3$. We remark that $c_{3,0}=0$. For each $m$ and any $r \geq 0$, there exists a family $\left\{u_{j, m, r, q}\right\}_{q \in \mathbb{N}}$ of compactly supported smooth functions on $V$ such that the following conditions are satisfied.

(5.3) For any compact subset of $V$, if we take $q$ sufficiently large, then the support of $u_{j, m, r, q}$ lies outside this compact set.

(5.4) For some positive constant $C_{15}$ independent of $q$, we have

$$
\left\|\left(\Delta-\left(c_{j, m}+r^{2}\right)\right) u_{j, m, r, q}\right\| \leq C_{15} \frac{1}{q}\left\|u_{j, m, r, q}\right\| .
$$

Let $\varphi$ be an eigenfunction belonging to the eigenvalue $c_{3, m}$. Then we can make a family of compactly supported smooth functions on $\hat{Y}_{1}$ (resp. $\hat{Y}_{2}$ ) satisfying the similar conditions as (5.3), (5.4) by applying Theorem 1 (2). Then, from Proposition 1.2, we can make two families of compactly supported smooth functions on $V$ satisfying the similar conditions as (5.3), (5.4) out of these families. On the other hand, we may suppose that $\left\{u_{3, m, r, q}\right\}_{q \in \mathbb{N}}$ is made of $\varphi$. Consequently, the eigenfunction $\varphi$ seems to produce three different Weyl sequences on $V$. However, if we use parallelograms instead of the disks $\mathcal{D}, \mathcal{D}^{\prime}$ in $\mathbb{R}^{2}$ in the proof of Theorem 1 , these are essentially the same. Such a phenomenon is already observed in the case of higher $\mathbb{Q}$-rank locally symmetric spaces. 


\section{Acknowledgement}

The author would like to thank Werner Müller, Pierre Pansu and Toshiyuki Kobayashi for their interest, suggestions, and encouragement from the early stage of this work. He would also like to thank the referee for carefully reading the manuscript and for many helpful suggestions.

\section{References}

[1] T. Aubin, Espaces de Sobolev sur les variétés riemanniennes, Bull. Sci. Math. (2) 100 (1976), no. 2, 149-173.

[2] W. Ballmann, M. Gromov and V. Schroeder, Manifolds of nonpositive curvature, Progr. Math., 61, Birkhäuser Boston, Boston, MA, 1985.

[3] A. Borel, Linear algebraic groups, Second edition, Graduate Texts in Math., 126, Springer, New York, 1991.

[4] - Introduction aux groupes arithmétiques, Hermann, Paris, 1969.

[5] Stable real cohomology of arithmetic groups, Ann. Sci. École Norm. Sup. (4) 7 (1974), 235-272.

[6] A. Borel and H. Garland, Laplacian and the discrete spectrum of an arithmetic group, Amer. J. Math. 105 (1983), no. 2, 309-335.

[7] A. Borel and J.-P. Serre, Corners and arithmetic groups, Comment. Math. Helv. 48 (1973), 436-491.

[8] P. R. Chernoff, Essential self-adjointness of powers of generators of hyperbolic equations, J. Funct. Anal. 12 (1973), 401-414.

[9] H. Donnelly, On the point spectrum for finite volume symmetric spaces of negative curvature, Comm. Partial Differential Equations 6 (1981), no. 9, 963-992.

[10] H. Donnelly and P. Li, Pure point spectrum and negative curvature for noncompact manifolds, Duke Math. J. 46 (1979), no. 3, 497-503.

[11] P. B. Eberlein, Geometry of nonpositively curved manifolds, Chicago Lectures in Math., Univ. Chicago Press, Chicago, IL, 1996.

[12] Lattices in spaces of nonpositive curvature, Ann. of Math. (2) 111 (1980), no. 3, $435-476$.

[13] M. P. Gaffney, The harmonic operator for exterior differential forms, Proc. Nat. Acad. Sci. U. S. A. 37 (1951), 48-50.

[14] H. Garland and M. S. Raghunathan, Fundamental domains for lattices in (R-)rank 1 semisimple Lie groups, Ann. of Math. (2) 92 (1970), 279-326.

[15] I. M. Glazman, Direct methods of qualitative spectral analysis of singular differential operators, Translated from the Russian by the IPST staff, Israel Program for Scientific Translations, Jerusalem, 1965; Daniel Davey \& Co., Inc., New York, 1966.

[16] T. Hattori, Geometry of quotient spaces of $\mathrm{SO}(3) \backslash \mathrm{SL}(3, \mathbf{R})$ by congruence subgroups, Math. Ann. 293 (1992), no. 3, 443-467.

[17] Collapsing of quotient spaces of $\mathrm{SO}(n) \backslash \mathrm{SL}(n, \mathbf{R})$ at infinity, J. Math. Soc. Japan 47 (1995), no. 2, 193-225.

[18] Asymptotic geometry of arithmetic quotients of symmetric spaces, Math. Z. 222 (1996), no. 2, 247-277.

[19] S. Lang, Fundamentals of differential geometry, Graduate Texts in Math., 191, Springer, New York, 1999.

[20] R. P. Langlands, On the functional equations satisfied by Eisenstein series, Lecture Notes in Math., 544, Springer, Berlin, 1976.

[21] P. D. Lax and R. S. Phillips, Scattering theory for automorphic functions, Ann. of Math. Stud., 87, Princeton Univ. Press, Princeton, N.J., 1976. 
[22] E. Leuzinger, An exhaustion of locally symmetric spaces by compact submanifolds with corners, Invent. Math. 121 (1995), no. 2, 389-410.

[23] W. Müller, Manifolds with cusps of rank one, Lecture Notes in Math., 1244, Springer, Berlin, 1987.

[24] , On the $L^{2}$-index of Dirac operators on manifolds with corners of codimension two. I, J. Differential Geom. 44 (1996), no. 1, 97-177.

[25] M. S. Osborne and G. Warner, The theory of Eisenstein systems, Academic Press, New York, 1981.

[26] G. Prasad, Strong rigidity of Q-rank 1 lattices, Invent. Math. 21 (1973), 255-286.

[27] M. S. Raghunathan, A note on quotients of real algebraic groups by arithmetic subgroups, Invent. Math. 4 (1967/1968), 318-335.

[28] L. Saper, Tilings and finite energy retractions of locally symmetric spaces, Comment. Math. Helv. 72 (1997), no. 2, 167-202.

[29] G. Warner, Harmonic analysis on semi-simple Lie groups. I, Springer, New York, 1972.

[30] R. J. Zimmer, Ergodic theory and semisimple groups, Birkhäuser, Basel, 1984.

[31] S. Zucker, $L_{2}$-cohomology and intersection homology of locally symmetric varieties. II, Compositio Math. 59 (1986), no. 3, 339-398. 